\title{
An integrated multiomic and quantitative label-free microscopy- based approach to study pro-fibrotic signalling in ex vivo human precision-cut lung slices
}

\author{
Muzamil Majid Khan $\mathbb{1}^{1,2,3}$, Daniel Poeckel ${ }^{2}$, Aliaksandr Halavatyi $\mathbb{1}^{1,3}$, Joanna Zukowska-Kasprzyk ${ }^{1}$, \\ Frank Stein ${ }^{1}$, Johanna Vappiani ${ }^{2}$, Daniel C. Sevin ${ }^{2}$, Christian Tischer ${ }^{1}$, Nico Zinn ${ }^{2}$, Jessica D. Eley ${ }^{4}$, \\ Natasja Stæhr Gudmann ${ }^{5}$, Thomas Muley ${ }^{3,6}$, Hauke Winter ${ }^{3,6}$, Andrew J. Fisher ${ }^{7}$, Carmel B. Nanthakumar ${ }^{4}$, \\ Giovanna Bergamini $\mathbb{1}^{2,8}$ and Rainer Pepperkok ${ }^{1,3,8}$
}

\begin{abstract}
${ }^{1}$ European Molecular Biology Laboratory, Heidelberg, Germany. ${ }^{2}$ Discovery Biology, Cellzome GmbH, GSK, Heidelberg, Germany. ${ }^{3}$ Lung Research Center Heidelberg (TLRC), German Center for Lung Research (DZL), Heidelberg, Germany. ${ }^{4}$ Novel Human Genetics Research Unit, GSK, Stevenage, UK. ${ }^{5}$ Nordic Bioscience, Herlev, Denmark. ${ }^{6}$ Biobank Thoraxklinik, University Hospital Heidelberg, Heidelberg, Germany. ${ }^{7}$ Newcastle University Translational and Clinical Research Institute and Institute of Transplantation, Newcastle upon Tyne Hospitals, Newcastle upon Tyne, UK. ${ }^{8} \mathrm{G}$. Bergamini and R. Pepperkok contributed equally to this article as lead authors and supervised the work.
\end{abstract}

Corresponding author: Rainer Pepperkok (pepperko@embl.de)

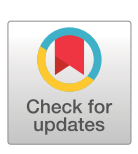

Copyright @ERS 2021.

This version is distributed under the terms of the Creative Commons Attribution Non-Commercial Licence 4.0. For commercial reproduction rights and permissions contact permissions@ersnet.org

This article has supplementary material available from erj.ersjournals.com

This article has an editorial commentary: https://doi.org/10. $1183 / 13993003.00203-2021$

Received: 3 Feb 2020 Accepted: 9 Dec 2020

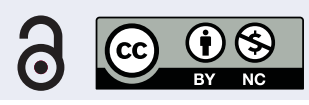

Shareable abstract (@ERSpublications)

Multiomic and label-free imaging-based characterisation of ex vivo cultured human precision-cut lung slices (hPCLS) reveals that MMP signalling is a rate-limiting factor necessary for deposition of fibrillar collagen in ECM of hPCLS https://bit.ly/3rcUa0e

Cite this article as: Khan MM, Poeckel D, Halavatyi A, et al. An integrated multiomic and quantitative label-free microscopy-based approach to study pro-fibrotic signalling in ex vivo human precision-cut lung slices. Eur Respir J 2021; 58: 2000221 [DOI: 10.1183/13993003.00221-2020].

\section{Abstract}

Fibrosis can affect any organ, resulting in the loss of tissue architecture and function with often lifethreatening consequences. Pathologically, fibrosis is characterised by the expansion of connective tissue due to excessive deposition of extracellular matrix (ECM) proteins, including the fibrillar forms of collagen. A significant limitation for discovering cures for fibrosis is the availability of suitable human models and techniques to quantify mature fibrillar collagen deposition as close as possible to human physiological conditions.

Here we have extensively characterised an ex vivo cultured human lung tissue-derived, precision-cut lung slices (hPCLS) model using label-free second harmonic generation (SHG) light microscopy to quantify fibrillar collagen deposition and mass spectrometry-based techniques to obtain a proteomic and metabolomic fingerprint of hPCLS in ex vivo culture.

We demonstrate that hPCLS are viable and metabolically active, with mesenchymal, epithelial, endothelial and immune cell types surviving for at least 2 weeks in ex vivo culture. Analysis of hPCLS-conditioned supernatants showed a strong induction of pulmonary fibrosis-related ECM proteins upon transforming growth factor- $\beta 1$ (TGF- $\beta 1$ ) stimulation. This upregulation of ECM proteins was not translated into an increased deposition of fibrillar collagen. In support of this observation, we revealed the presence of a pro-ECM degradation activity in our ex vivo cultures of hPCLS, inhibition of which by a metalloproteinase inhibitor resulted in increased collagen deposition in response to TGF- $\beta 1$ stimulation.

Together the data show that an integrated approach of measuring soluble pro-fibrotic markers alongside quantitative SHG-based analysis of fibrillar collagen is a valuable tool for studying pro-fibrotic signalling and testing anti-fibrotic agents.

\section{Introduction}

Excessive deposition of extracellular matrix (ECM) proteins is a hallmark of fibrosis. It alters tissue architecture and results in loss of function and, ultimately, end-stage organ failure [1]. In the developed world, $45 \%$ of all deaths are attributed to conditions related to an excess of ECM deposition [2]. While 
fibrosis contributes to exacerbated pathology in cancer, myocardial infarctions and ageing, it is also a primary cause of mortality in fibrotic disease of the kidneys, liver and lung in particular [3]. Chronic interstitial lung diseases (ILDs) are the most common form of pulmonary fibrosis [4]. ILDs have been divided into over 300 subtypes, among which idiopathic pulmonary fibrosis (IPF) is the most prevalent [4]. Damage to epithelial and endothelial cells in various organs launches an inflammatory response, which triggers blood clot formation and ECM repair. Part of this repair mechanism is the release of cytokines such as transforming growth factor- $\beta 1$ (TGF- $\beta 1$ ) that initiate the activation of macrophages and fibroblasts [3]. Activated fibroblasts express $\alpha$-smooth muscle actin ( $\alpha$-SMA), which leads to their differentiation in myofibroblasts [3]. Persistent chronic inflammation triggers unchecked proliferation of myofibroblasts, increasing epithelial-to-mesenchymal transition (EMT) [3]. The mesenchymal population of cells has an enhanced ability to produce ECM components, and the combination of these events results in a derailed tissue repair process leading to tissue scarring [3]. TGF- $\beta 1$ is considered to be the master regulator of the signalling pathways (pro-fibrotic) that lead to an abnormally excessive deposition of ECM [5]. Induction of pro-fibrotic signalling also shifts the delicate balance between synthesis and break down of collagens and other ECM proteins [6] in favour of excessive deposition and pathological stiffening of ECM with loss of tissue compliance. This excessive deposition and stiffness of ECM causes a physical abnormality that leads to alveolar tissue atrophy [3] and subsequent suffocation (dyspnoea). In IPF patients, the fibrotic foci are characterised by an abnormally high content of fibrillar collagen (type I, III and V) [3, 7]. At the ECM level, presence of these fibrillar collagens is the physical cause of eventual mortality [8]. Despite our understanding of the molecular basis underlying the fibrotic process, to date only two licensed drugs, pirfenidone and nintedanib [9], have been approved for the treatment of IPF. These medicines have very low efficacy and, hence, high patient withdrawal rates [10]. The lack of more effective anti-fibrotic therapies may be attributed in part to the absence of adequate model systems that can mimic the pathophysiology of the fibrotic process as related to human physiology [11], and to indirect functional readouts of biomarkers used for quantifying disease progression. Currently, murine experimental models of fibrosis are frequently used to study lung, liver and kidney fibrosis. Typically, pulmonary fibrosis is induced either using genetic manipulation such as overexpressing mucin 5b (MUC5b), a genetic risk factor identified in large cohorts of IPF patients [12], or chemical induction (bleomycin sulfate, amiodarone, carbon tetrachloride) [13]. These model systems help to elucidate acute signalling pathways preceding tissue damage and, in some instances, chemicals such as bleomycin sulfate can be used as causative agents [14]; however, in vivo models lack histological features of human disease and can spontaneously resolve, and they therefore fail to recapitulate the irreversible end-stage disease. These model systems have highlighted an inflammatory component present during the development of fibrotic disease, yet anti-inflammatory drugs have failed in clinical development $[15,16]$. Based on these findings, therapies involving corticosteroids (prednisone) with or without immunosuppressive drugs (azathioprine and cyclophosphamide) have been used to treat IPF. In fact, some drugs have had adverse effects that worsen patient condition $[15,16]$.

Human precision-cut lung slices (hPCLS) are three-dimensional (3D), uniformly cut slices of lung tissue that may be maintained in ex vivo culture. They have emerged as a promising model system to study human disease in vitro. The use of PCLS (rat lung and human) was first reported in the early 1990s [17, 18] but they have only recently been used as a model for evaluating human disease pathophysiology [11, 19]. Several groups have used this model system to recapitulate pro-fibrotic signalling [11, 19, 20]. Recently, AKram et al. [21] studied the dynamics of epithelial cells in mouse PCLS. However, the overall molecular changes and changes to molecular pathways in ex vivo culture of human PCLS are still elusive. Earlier studies $[11,19]$ do show an induction in fibrotic signalling, but a functional readout of enhanced ECM deposition has been lacking. Furthermore, whether cell types essential for fibrosis such as epithelial, mesenchymal and immune cell types [3] are present in the model system has not been investigated. Although it is generally assumed that cell-cell and cell-matrix interactions are well preserved in hPCLS [21], no systematic study has been conducted to evaluate such a hypothesis.

ELISA-based measurements can be used to quantify the cleavage products of collagen propeptides, such as type I, III and VI collagen, because they are cleaved off during formation. These cleavage products provide information on fibrosis disease progression [22]. While they could prove to be disease biomarkers, their correlation with excess deposition is still not established. Microscopic detection of fibrillar collagen is also difficult owing to the lack of specific antibodies. However, label-free second harmonic generation (SHG) imaging for fibrillar collagen is an excellent alternative method for quantifying fibrillar collagen in tissue slices [23].

In this study, we used mass spectrometry (MS) and light microscopy-based approaches to first investigate and characterise the changes of protein expression in the four major cell types present in lung tissue when 
hPCLS are ex vivo cultured. Second, to investigate the pro-fibrotic signalling induced by TGF- $\beta 1$ stimulation, we established a new, quantitative, label-free SHG imaging approach that quantifies deposited fibrillar collagen in the ECM upon pro-fibrotic stimulation. We propose that this model represents a versatile human translational model system that could be used for identifying and characterising anti-fibrotic agents.

Materials and methods

Lung resection supply and licences

All the hPCLS that were cultured ex vivo were derived from lung tissues resected from tumour-free areas (26 donors). Donor demographics, including age, sex, smoking status, major clinical diagnosis and the

TABLE 1 Patient information

\begin{tabular}{|c|c|c|c|c|c|}
\hline $\begin{array}{r}\text { Anonymised } \\
\text { patient ID }\end{array}$ & $\begin{array}{l}\text { Age } \\
\text { years }\end{array}$ & Sex & $\begin{array}{l}\text { Smoking } \\
\text { status }\end{array}$ & Disease diagnosis & Tissue used \\
\hline LT04 & 66 & $\mathrm{~F}$ & $\mathrm{~S}$ & Adenocarcinoma & Resection \\
\hline LT05 & 68 & M & $\mathrm{S}$ & Squamous cell carcinoma & Resection \\
\hline LT06 & 78 & $\mathrm{~F}$ & ES & Squamous cell carcinoma & Resection \\
\hline LT07 & 50 & M & ES & Adenocarcinoma & Resection \\
\hline LT08 & 63 & M & $\mathrm{S}$ & Squamous cell carcinoma & Resection \\
\hline LT13 & 48 & M & $\mathrm{N}$ & Metastasis & Resection \\
\hline LT18 & 67 & $\mathrm{~F}$ & $\mathrm{~S}$ & Adenocarcinoma & Resection \\
\hline LT19 & 60 & $\mathrm{~F}$ & ES & Carcinoma & Resection \\
\hline LT21 & 56 & $\mathrm{~F}$ & $\mathrm{~S}$ & Adenocarcinoma & Resection \\
\hline LT22 & 75 & $\mathrm{~F}$ & ES & Adenocarcinoma & Resection \\
\hline LT24 & 68 & M & ES & Squamous cell carcinoma & Resection \\
\hline LT25 & 65 & M & $\mathrm{N}$ & Neuroendocrine tumour & Resection \\
\hline LT30 & 77 & $\mathrm{~F}$ & ES & Adenocarcinoma & Resection \\
\hline LT31 & 65 & M & ES & Squamous cell carcinoma & Resection \\
\hline LT32 & 68 & $\mathrm{~F}$ & ES & Squamous cell carcinoma & Resection \\
\hline LT35 & 69 & M & ES & Adenocarcinoma & Resection \\
\hline LT36 & 62 & M & ES & Squamous cell carcinoma & Resection \\
\hline LT37 & 63 & $\mathrm{~F}$ & $\mathrm{~S}$ & Adenocarcinoma & Resection \\
\hline LT38 & 67 & M & $\mathrm{S}$ & $\begin{array}{c}\text { Nonsmall-cell lung } \\
\text { carcinoma }\end{array}$ & Resection \\
\hline LT39 & 67 & $\mathrm{~F}$ & $\mathrm{~S}$ & $\begin{array}{l}\text { Nonsmall-cell lung } \\
\text { carcinoma }\end{array}$ & Resection \\
\hline LT40 & 68 & $\mathrm{~F}$ & $\mathrm{~N}$ & Adenocarcinoma & Resection \\
\hline LT44 & 68 & M & $\mathrm{S}$ & Squamous cell carcinoma & Resection \\
\hline LT62 & 50 & M & ES & Carcinoma & Resection \\
\hline LT63 & 67 & M & ES & Adenocarcinoma & Resection \\
\hline LT139 & 69 & M & $\mathrm{S}$ & Adenocarcinoma & Resection \\
\hline L340 & 62 & M & ES & IPF & $\begin{array}{l}\text { Transplant } \\
\text { recipient }\end{array}$ \\
\hline L348 & 59 & M & ES & IPF & $\begin{array}{l}\text { Transplant } \\
\text { recipient }\end{array}$ \\
\hline L324 & 59 & M & $\mathrm{S}$ & NSIP & $\begin{array}{l}\text { Transplant } \\
\text { recipient }\end{array}$ \\
\hline L323 & 58 & M & $\mathrm{N}$ & IPF & $\begin{array}{l}\text { Transplant } \\
\text { recipient }\end{array}$ \\
\hline L315 & 56 & M & $\mathrm{N}$ & IPF & $\begin{array}{l}\text { Transplant } \\
\text { recipient }\end{array}$ \\
\hline ET01 & 68 & M & ES & Squamous cell carcinoma & Resection \\
\hline ET02 & 68 & $\mathrm{~F}$ & $\mathrm{~N}$ & Adenocarcinoma & Resection \\
\hline ET03 & 57 & $\mathrm{~F}$ & $\mathrm{~N}$ & Adenocarcinoma & Resection \\
\hline ET04 & 65 & $\mathrm{~F}$ & ES & Adenocarcinoma & Resection \\
\hline ET05 & 70 & $\mathrm{~F}$ & ES & Squamous cell carcinoma & Resection \\
\hline ET06 & 57 & M & ES & Squamous cell carcinoma & Resection \\
\hline
\end{tabular}

ID: identification; M: male; F: female; S: active smoker; N: never-smoker; ES: ex-smoker; IPF: idiopathic pulmonary fibrosis; NSIP: nonspecific interstitial pneumonia; Resection: tumour-free lung tissue (non-interstitial lung disease patients) from an area distant to lung tumour tissue resected; Transplant recipient: fibrotic lung tissue from fibrosis patients receiving a transplant. 
experiments the tissue was used in, are listed in table 1. Tumour-free tissue from lung tissue resections of lung cancer patients were obtained from Thoraxklinik-Heidelberg with anonymised patient identities. The tumour-free tissue was selected by the pathologist during tissue resection. Patient consent and use of tissue were obtained as per the research ethics committee (Medical Faculty of University Heidelberg) approval reference number S-270/2001. In addition, hPCLS were generated from ILD lung tissue obtained from the lung parenchyma of patients undergoing lung transplant for end-stage disease at the Institute of Transplantation, Newcastle upon Tyne Hospitals, UK (five lung transplant recipients; details in table 1). Fibrotic foci were not visible during the processing procedure; therefore, it was not possible to say whether the individual slices generated for each donor contained fibrotic foci. Patient consent and use of ILD tissue were approved by the National Research Ethics Service (11/NE/0291) and UK Health Research Authority. Human biological samples were sourced ethically and their research use was in accordance with the terms of informed consent under an institutional review board/ethics committee-approved protocol.

\section{PCLS preparation and ex vivo culture}

The overall workflow followed for this study is summarised in figure 1. Non-ILD and ILD hPCLS were prepared as described previously [24, 25]. Briefly, on the day the tissue resection sample was received (day 0), lung tissue was inflated with 3\% low melting agarose (Sigma \#A9414) prepared in phenol-free DMEM (Gibco \#41965-039). Next, $8 \mathrm{~mm}$ cores were prepared and 250- $\mu \mathrm{m}$-thick slices were generated using a Krumdieck tissue slicer (TSE Systems, Bad Homburg, Germany). To facilitate recovery following the slicing procedure, the tissue medium was supplemented with penicillin, streptomycin, fungizone and $10 \%$ FCS for the first $18 \mathrm{~h}$ of ex vivo culture. After $18 \mathrm{~h}$ (day 1) and then every $72 \mathrm{~h}$ till day 13, the media ( \pm stimulation) was replenished. Cell culture-grade plastic 24-well plates (Greiner \#353226) were used to culture hPCLS. On day 0 medium was additionally supplemented with 10\% FCS (Gibco \#10270106). The hPCLS were treated with $10 \mathrm{ng} \cdot \mathrm{mL}^{-1}$ recombinant human TGF- $\beta 1$ protein (R\&D Systems \#240-B-010) and $25 \mu \mathrm{M}$ (in $750 \mu \mathrm{L}$ of DMEM) of matrix metalloproteinase (MMP) inhibitor (MMPi) (Merck \#CC1010). Live/dead staining was carried out using a calcein-AM/ethidium homodimer kit (ThermoFisher \#L3224) as per supplier recommendations. The hPCLS were incubated in the recommended concentration of live/dead reagents for $45 \mathrm{~min}$ prior to live confocal microscopy. hPCLS were always cultured in the presence of penicillin-streptomycin (Gibco \#15140122) and amphotericin B (Gibco \#15290026).

\section{ELISA measurements}

Soluble ECM fragments (procollagen 1 N-terminal propeptide (PRO-C1), C-terminal part of fibronectin (FBN-C) and collagen type III degradation marker (C3M)) were determined in the media supernatant by competitive (ELISA) assays developed and validated by Nordic Bioscience A/S (Herlev, Denmark) according to the manufacturer's instructions [26-28]. Values measured below the detection limit of the assay were assigned the lower limit of detection.

\section{MS sample preparation and analysis}

Proteomics

Briefly, hPCLS were washed with PBS and harvested using snap freezing in liquid nitrogen. Upon thawing, samples (two hPCLS per technical replicate) were dissolved in $300 \mu \mathrm{L}$ of $2 \%$ SDS- $\mathrm{H}_{2} \mathrm{O}$ (with protease and phosphatase inhibitor) for $2 \mathrm{~h}$ at room temperature. They were then mechanically homogenised using a bead ruptor (Omni International \#19-040). Before liquid chromatography with tandem MS (LC-MS/MS) analysis, protein concentrations were measured (Pierce $660 \mathrm{~nm}$ kit \#22660). Samples were solubilised in $2 \times$ SDS sample buffer and subjected to short SDS gel electrophoresis. Samples were further processed for LC-MS/MS analysis.

SDS-PAGE gels were Coomassie stained. Gel lanes were cut into three slices covering the entire separation range $(\sim 2 \mathrm{~cm})$ and subjected to in-gel tryptic digestion [29]. Peptides were labelled via isobaric mass tags (TMT10; Thermo Fisher Scientific).TMT labelling was performed using the 10-plex TMT reagents, enabling relative quantification of 10 conditions in a single experiment [30]. Briefly, the labelling reaction was performed in $40 \mathrm{mM}$ triethylammonium bicarbonate, $\mathrm{pH} 8.53$ at $22^{\circ} \mathrm{C}$, and quenched with glycine. Labelled peptide extracts were combined to a single sample per experiment. Lyophilised samples were re-suspended in $1.25 \%$ ammonia in water and subjected to LC-MS/MS [30].

\section{Peptide and protein identification}

Mascot 2.4 (Matrix Science, Boston, MA, USA) was used for protein identification using a 10 parts per million mass tolerance for peptide precursors and $20 \mathrm{mD}$ higher-energy collisional dissociation (HCD) mass tolerance for fragment ions. The search database consisted of a customised version of the International Protein Index protein sequence database combined with a decoy version of this database created using scripts supplied by MatrixScience. 

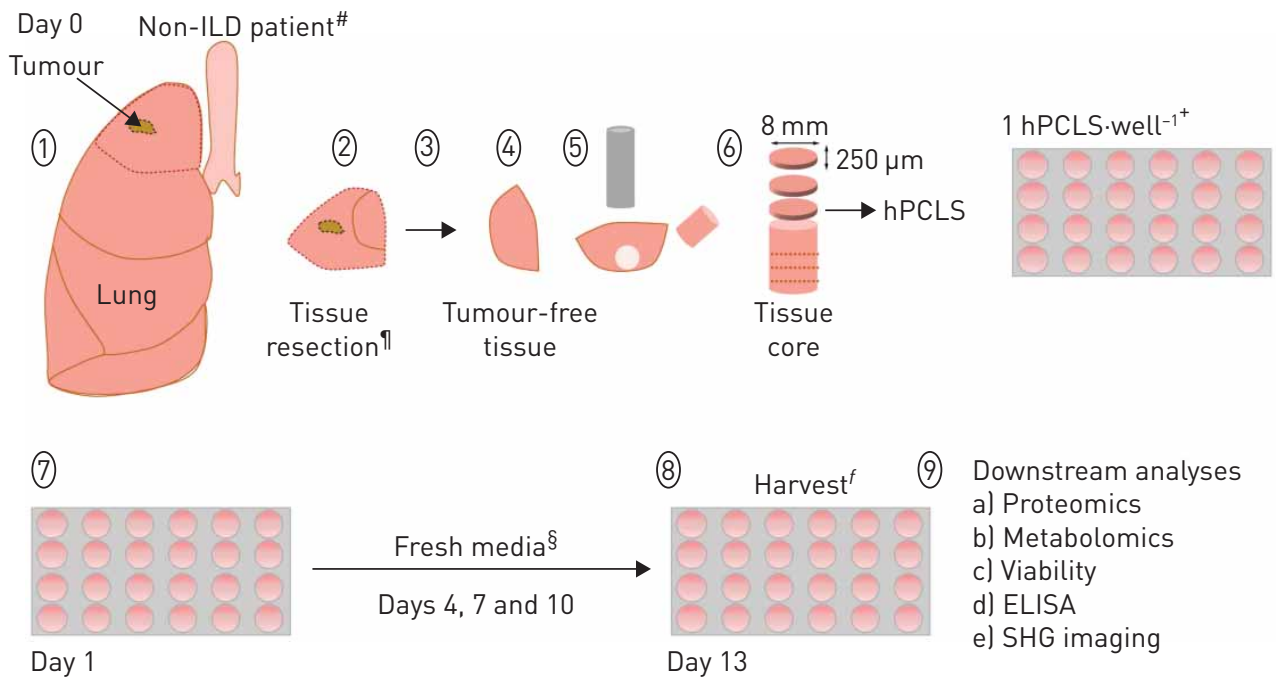

FIGURE 1 Schematic representation of the experimental workflow. 1-3: On day 0, tumour-free tissue (distal to tumour site) resected from patients with non-interstitial lung disease (ILD) was surgically removed and classified as tumour-free by the local pathologist. 4: Tumour-free tissue was received and inflated with $3 \%$ low melting agarose. 5: Tissue cores of varying heights were prepared. 6: Human precision-cut lung slices (hPCLS) of $8 \mathrm{~mm}$ diameter and $250 \mu \mathrm{m}$ thickness were prepared using a Krumdieck tissue slicer. On day 0, each hPCLS generated was distributed across a 24-well plate $\left(1 \mathrm{hPCLS} \cdot\right.$ well $\left.^{-1}\right)$. For all the days of culture, each hPCLS was cultured in $750 \mu \mathrm{L}$ of DMEM with constant presence of antimicrobials (amphotericin B and penicillinstreptomycin). From day 0 to day $1(18 \mathrm{~h}$ ), the tissue was kept in DMEM with $10 \%$ FCS. 7 : On day 1, old media (with FCS) was replaced with fresh media (no FCS). Media (with no FCS) was also replenished on days 4, 7 and 10. When required, hPCLS were additionally treated with transforming growth factor- $\beta 1$ (TGF- $\beta 1$ ) or any other stimulant from day 1 onwards. 8-9: On day 13, hPCLS were harvested for downstream second harmonic generation (SHG) analysis and proteomics (involving TGF- $\beta 1$ stimulation). \#: for SHG analysis only (non-ILD versus ILD), five ILD patient-derived hPCLS were used, which were PFA fixed directly on day 0; ฯ: for ILD patients, the tissue was taken from the lung parenchyma of transplant-recipient patients; ${ }^{+}:$hPCLS were treated with DMEM containing $10 \%$ FCS only for first $18 \mathrm{~h}$ of culture; ${ }^{\S}$ : in experiments involving ELISA measurements, media supernatants (days 4,7 and 13 ) from each hPCLS ( \pm TGF- $\beta 1$ ) were collected separately and subjected to soluble marker measurements; ${ }^{f}$ : for proteomics and metabolomics of hPCLS without any treatment, slices were also harvested on days $1,4,7$ and 10 in addition to day 13 and for live imaging viability analysis on days 1,7 and 13 .

Reporter ion intensities were read from raw data and multiplied with ion accumulation times (in ms) to yield a measure proportional to the number of ions; this measure is referred to as ion area [31]. Spectra matching peptides were filtered according to the following criteria: mascot ion score $>15$, signal-to-background of the precursor ion $>4$ and signal-to-interference $>0.5$ [31]. Fold changes were corrected for isotope purity as described and adjusted for interference caused by co-eluting nearly isobaric peaks as estimated by the signal-to-interference measure [31]. Protein quantification was derived from individual spectra matching to distinct peptides using a sum-based bootstrap algorithm; 95\% confidence intervals were calculated for all protein fold changes that were quantified with more than three spectra [31].

\section{Metabolomics}

hPCLS were snap-frozen on the day of harvest. On the day of sample preparation, two hPCLS per technical replicate were briefly rinsed in $75 \mathrm{mM}$ ammonium bicarbonate ( $\mathrm{pH}$ 7.4) and mechanically homogenised in MS-grade $\mathrm{H}_{2} \mathrm{O}$ to extract metabolites. Untargeted metabolomics analysis was performed as previously described [32]. Briefly, samples were analysed on a liquid chromatography-mass spectrometry (LC/MS) platform consisting of a Thermo Scientific Ultimate 3000 LC system with autosampler temperature set to $10^{\circ} \mathrm{C}$ coupled to a Thermo Scientific Q-Exactive Plus Fourier transform MS equipped with a heated electrospray ion source and operated in negative ionisation mode. The isocratic flow rate was $150 \mu \mathrm{L} \cdot \mathrm{min}^{-1}$ of mobile phase consisting of 60:40\% (v/v) isopropanol:water buffered with $1 \mathrm{mM}$ ammonium fluoride at $\mathrm{pH} 9$ and containing $10 \mathrm{nM}$ taurocholic acid and $20 \mathrm{nM}$ homotaurine as lock masses. Mass spectra were recorded in profile mode from 50 to $1000 \mathrm{~m} \cdot \mathrm{z}^{-1}$ with the following instrument 
settings: sheath gas, 35 a.u.; auxiliary gas, 10 a.u.; auxiliary gas heater, $200^{\circ} \mathrm{C}$; sweep gas, 1 a.u.; spray voltage, $-3 \mathrm{kV}$; capillary temperature, $250^{\circ} \mathrm{C}$; S-lens radio frequency level, 50 a.u.; resolution, $70 \mathrm{k}$ at $200 \mathrm{~m} \cdot \mathrm{z}^{-1}$; automatic gain control target, $3 \times 10^{6}$ ions, maximum injection time, $120 \mathrm{~ms}$; acquisition duration, $60 \mathrm{~s}$. Spectral data processing was performed using an automated pipeline in R (www.r-project. org) as described previously [32]. Detected ions were tentatively annotated as metabolites based on matching accurate mass within a tolerance of $5 \mathrm{mDa}$ using the Human Metabolome database [33].

\section{Data analysis}

Proteomics

The R programming language (www.r-project.org) was employed to process the protein output files of IsobarQuant. Only proteins that were quantified with at least two unique peptides and that were quantified in all experiments were used for further analysis. The "sumionarea protein" columns were annotated to different experimental conditions. Then, potential batch effects were removed using the limma package [34] and the results were normalised using the vsn package [35]. Limma was also used to test for differential expression of proteins. A protein was considered significant with a two-fold difference and a false discovery rate $<5 \%$. All significant proteins were clustered (hierarchical clustering using the ward.d2 method) based on their Euclidean distances of log2 ratios towards the respective control.

Network analysis using Cytoscape

Network analysis was carried out using open-source software platform Cytoscape [36]. Briefly, genes that were significantly regulated (log2 fold change (FC) $\geqslant 0.58$ or $\leqslant 0.58$ and adjusted p-value $\leqslant 0.05$ ) were curated and subjected to pathway analysis. The graphs were plotted using an in-house R script.

\section{Lung Gene Expression Analysis-based analysis for cell types}

The list of genes for major cell types was downloaded from the "LungSortedCells" tool on the Lung Gene Expression Analysis (LGEA) database. Here, RNA-sequencing (RNA-seq) data defined as belonging to the category "adult" was used as a reference. These data were generated after RNA-seq of sorted cells as per the following markers: CD45 ${ }^{+}$immune cells, CD45 $/ \mathrm{PECAM}^{-} / \mathrm{VECadherin}^{-} / \mathrm{EpCAM}^{+}$mixed epithelial cells, CD $45^{-} / \mathrm{PECAM}^{+} / \mathrm{VECadherin}^{+}$mixed endothelial cells and $\mathrm{CD}^{-} 5^{-} / \mathrm{PECAM}^{-} / \mathrm{VECadherin}^{-} /$ $\mathrm{EpCAM}^{-}$mixed mesenchymal cells.

The curation of commonly used lung cell-type markers was done using the LGEA Tool-Box feature. Here, LungGENS Human RNA-seq data classified under "Human-Dropseq-PND1" was used as reference.

\section{SHG imaging and sample preparation}

On the day of harvest, the medium supernatant was aspirated from each well containing hPCLS. hPCLS were washed with PBS $(3 \times 1 \mathrm{~mL})$ and fixed in $1 \mathrm{~mL}$ of $4 \%$ paraformaldehyde (PFA) (Electron Microscopy Services; EM grade \#5710) overnight at $4^{\circ} \mathrm{C}$. Subsequently, hPCLS were washed with PBS $(3 \times 1 \mathrm{~mL})$. Next, each PFA-fixed hPCLS was transferred to a 24-well plate with a glass bottom (Greiner Bio-One International SensoPlate \#662892). hPCLS were covered with $150 \mu \mathrm{L}$ of PBS and pressed to the bottom using a circular glass cover slip and plastic ring (made locally at the EMBL workshop). Next, each well was covered with a further $300 \mu \mathrm{L}$ of PBS to avoid the hPCLS drying out (owing to PBS evaporation during image acquisition).

For the live imaging experiment, the space between the walls of the well and the hPCLS was filled with a thin layer of 5\% low melting agarose to prevent movement caused by changes in the medium over time in ex vivo culture.

SHG imaging of hPCLS was performed on a Zeiss NLO LSM780 microscope equipped with a pulsed multiphoton laser. hPCLS were excited with a two-photon wavelength of $880 \mathrm{~nm}$ with a $\times 20(0.8$ numerical aperture) air objective and a $\times 25$ (0.8 numerical aperture) water objective for imaging PFA-fixed and live hPCLS, respectively. 150-180 $\mu$ m-deep z-stacks were acquired with a square tiled (7-8×7-8 mm) scan. Two-photon excited signals originating from hPCLS were captured in three different channels: backwards SHG, forwards SHG and two-photon excited autofluorescence (2PEA). The forwards SHG signal was captured using a laser blocking filter and a band pass filter of 436/20 nm. The backwards SHG signal was recorded using internal detectors in a bandwidth of $435-455 \mathrm{~nm}$. 2PEA was detected in a bandwidth of 550-650 $\mathrm{nm}$.

\section{Calcein-AM/ethidium homodimer-1 imaging and ATP-Glo assay}

A LIVE/DEAD Viability/Cytotoxicity Kit (ThermoFisher \#L3224) was used for calcein-AM/ethidium homodimer-1 imaging. On the day of imaging, live hPCLS were first washed with $\times 1$ phenol-free DMEM 
and then incubated (45 min to $1 \mathrm{~h}$ ) with $500 \mu \mathrm{L}$ of $1 \mu \mathrm{M}$ and $2 \mu \mathrm{M}$ working solution of calcein-AM and ethidium homodimer-1, respectively (at $37^{\circ} \mathrm{C}, 5 \% \mathrm{CO}_{2}$ ). Stained, live hPCLS were transferred into a $35 \mathrm{~mm}$ MatTek dish with a glass coverslip in the centre (\#D35-10-1.5-N). The hPCLS were generally imaged for 20-25 min in a moist MatTek chamber with $100 \mu \mathrm{L}$ of phenol-free DMEM (Sigma \#D2902) covering the hPCLS. After the imaging, hPCLS were discarded. On the subsequent day of imaging, new hPCLS were stained and imaged. A CellTiter-Glo Luminescent assay (Promega \#G7570) was used to measure ATP levels following the manufacturer's protocol. Briefly, one hPCLS was homogenised using a bead ruptor in $250 \mu \mathrm{L}$ of the CellTiter-Glo reagent and each hPCLS lysate was measured in triplicate in 96-well plates ( $50 \mu \mathrm{L}$ triplicates of each hPCLS lysate). In total, four hPCLS were analysed per donor.

\section{Statistical analysis of ELISA and SHG imaging data}

In general, all the data were normalised to the mean value of all the hPCLS measurements (per donor) in the respective control (defined as per the experiment). First, the raw values of SHGintensity (iSHG) from different stimulation or disease conditions were log2 transformed (Log2iSHG) and the mean iSHG of unstimulated hPCLS was calculated and log2 transformed (Log2iSHGc). Next, the log fold change was calculated as Log2SHGi=Log2iSHG-Log2iSHGc for each slice of the respective donor. Upon global normalisation, the Log2iSHG of each hPCLS was normalised to the Log2iSHGc of all unstimulated hPCLS across the respective stimulation or condition. Two-way ANOVA with Tukey's multiple comparisons test was performed to determine the significance of the differences between comparisons. Two factors selected were 1) differences in donor to donor number of slices and 2) respective treatment or disease condition. Shapiro-Wilk normality test was used to confirmed data normality.

\section{SHG image analysis}

Image analysis of z-stacks consisting of SHG channels (backwards and forwards) and 2PEA were analysed using a semi-automated Fiji [37] pipeline defined as Jython script (supplementary material). Briefly, all the channels were thresholded and the area or the number of pixels below the threshold (PBT) were counted for each channel. The integrated intensity of PBT was also calculated for each channel (Sumintensity). To measure the total fibrillar collagen content, Sumintensity of PBT of the backwards and forwards SHG signal were summed up and termed as the total fibrillar collagen content. A sum of the maximum projections of forwards and backwards SHG (two-dimensional) images was created and this image was divided into $8 \times 8$ equally sized regions of interest (ROIs). Each ROI was manually classified as interstitial collagen or non-interstitial collagen. Only ROIs of interstitial collagen were averaged per hPCLS SHG image. Each experiment has unstimulated and treated hPCLS SHG images. The Sumintensity of total collagen content pixels $\cdot \mathrm{ROI}^{-1}$ was normalised to the average SHG signal in unstimulated (average of all the ROIs) hPCLS of the respective donor. For each hPCLS SHG stack a minimum of 20 ROIs were analysed.

\section{Fiji code for interstitial and non-interstitial ROI selection}

Step by step documentation of the code and the code itself is available as a compressed zip file (supplementary material).

\section{Results}

Proteomic characterisation of molecular changes in hPCLS over time in ex vivo culture conditions When using hPCLS, in this case derived from tissue resected from non-ILD patients, to mimic a particular disease involving certain cell types and signalling pathways, it is imperative to characterise the model system in given ex vivo culture conditions. To this end, we used MS-based proteomics and untargeted metabolomics to monitor the hPCLS proteome and metabolome over time in ex vivo culture. Ex vivo cultured hPCLS (four donors, two replicates per donor, two hPCLS per replicate) were harvested on different days (day 1, 4, 7, 10 and 13 in culture) and snap-frozen in liquid nitrogen. Subsequently, lysates were prepared for MS-based proteomic and metabolomic analysis. For proteomic analysis, 2\% SDS soluble fractions of hPCLS were subjected to LC/MS analysis. Approximately 5500 proteins were detected across the four donors tested, of which 4288 proteins were in common (supplementary table S1). These proteins could be attributed to different cellular localisations bioinformatically using the Gene Ontology (GO) database [38]. The percentage of proteins per GO term was calculated relative to the whole GO term independent of its expression in human lung tissue. Here, 3648 proteins (31.75\%, reference list of 11488 proteins, GO: 0005737) were cytoplasmic, 2556 (25.9\%, reference list of 9856 proteins, GO: 0016020) were membrane related, 1149 (54.2\%, reference list of 2119 proteins, GO: 1903561) were extracellular vesicle related and 207 (38.6\%, reference list of 536 proteins, GO: 0031012) were ECM related. 187 proteins (45.7\%, reference list of 409 proteins, GO: 0062023) could be associated with the collagen-containing extracellular matrix GO term. (The number of proteins does not add up to 5500 because many of the proteins are classified as belonging to more than one GO term.) 
Differential analysis (figure 2a) of Log2FC in protein levels with respect to the previous time point of harvest shows that hPCLS undergo significant changes. On day 13, 728 (17\%, supplementary table S1) out of the 4288 proteins had changed significantly compared to day 1 . Of these, $10 \%$ changed from day 1 to day 4 and the remaining $7 \%$ from day 4 to day 7 . Between day 7 and 13 no further changes were observed. In total, 343 out of the 728 changing proteins were downregulated and 385 were upregulated.

Cytoscape-based network analysis (supplementary figure S2b) of the 728 proteins showed primarily an upregulation of pathways involving proteins related to ECM degradation. Fewer pathways were related to the formation of ECM. A significant upregulation of inflammatory signalling pathways, such as for neutrophil degranulation and the innate immune system, was also observed (supplementary figure S2b).

We further analysed the proteomics data to investigate the dynamics of different cell types underlying the observed changes in protein levels. Human lung tissue is mainly composed of immune, mesenchymal, endothelial and epithelial cells. The LGEA web portal has established a database of gene expression of each cell type (which is also age-dependent, from neonatal to adult stages) in both mouse and human lungs $[39,40]$. In this database, the RNA-seq of flow-sorted human lung cells have been designated as $\mathrm{CD} 5^{+}$immune cells, CD45 ${ }^{-} / \mathrm{PECAM}^{-} / \mathrm{VECadherin}^{-} / \mathrm{EpCAM}^{+}$mixed epithelial cells, $\mathrm{CD}^{-} 5^{-} / \mathrm{PECAM}^{+} /$ VECadherin ${ }^{+}$mixed endothelial cells and $\mathrm{CD} 45^{-} / \mathrm{PECAM}^{-} / \mathrm{VECadherin}^{-} / \mathrm{EpCAM}^{-}$mixed mesenchymal cells. The parent list can be downloaded from the LGEA database under "LungSortedCells". We compared (supplementary table S3a) significantly changing (Log2FC $\geqslant 0.5$ and $\leqslant-0.5$ and false discovery rate (FDR) $\mathrm{p} \leqslant 0.05$ ) proteins on day 13 versus day 1 to the list of proteins attributed to different cell classes (in LGEA [40] analysis). Supplementary figure S2c shows markers for four broadly categorised major cell types, namely, epithelial, endothelial, mesenchymal and immune cell types, which are typically present in lung tissue on day 13 in our culture system. Based on the LGEA database we also curated a list of commonly used lung cell-type markers (supplementary figure S2c, supplementary table S3). Of note, advanced glycosylation end-product specific receptor (AGER) (alveolar type 1) and surfactant protein C (SFTPC) (alveolar type 2) were both significantly downregulated in our ex vivo culture, while SFTPB, another alveolar type 2 marker, showed significant upregulation on day 13 compared to day 1 . The majority of the detectable myofibroblast markers showed a persistent decrease while fibroblast markers tenascin C (TNC) and platelet derived growth factor receptor- $\alpha$ (PDGFRA) showed an increase and vimentin (VIM) showed no change. Actin $\alpha 2$, smooth muscle (ACTA2), another well-established marker of myofibroblasts, could not be detected.

Among the significantly changing mesenchymal proteins, pro-fibrotic collagens [8] such as collagen type $\mathrm{V} \alpha 1$ chain (COL5A1) (Log2FC -0.7) and collagen type III $\alpha 1$ chain (COL3A1) (Log2FC -0.9) were significantly downregulated. Given that the collagen class of proteins is highly relevant for fibrosis progression [8], this observation prompted us to look into the pattern of regulation of all the collagens detected. The data show that under the culture conditions used, the majority of the detected collagens (figure 3) were downregulated over time, including COL1, COL4 and COL6. Furthermore, there was significant upregulation of MMP2 (Log2FC 2.1) and MMP14 (Log2FC 2.27); both these metalloproteinases are involved in degrading fibrillar collagens [41] such as COL1, COL2, COL3 and COL5. Two tissue inhibitors of metalloproteinases, TIMP2 and TIMP3 [42], were also detected (supplementary table S1). While TIMP3 did not show any major change (Log2FC -0.24, FDR 0.507, day 13 versus day 1), TIMP2 was upregulated over time (Log2FC 2.39, FDR $<0.0001$, day 13 versus day 1 ). In this context, it is important to note that TIMP2 has been shown to enhance the activity of MMP2 [43, 44], which is a collagen-degrading enzyme [45].

In general, the metalloproteinases detected over the course of ex vivo culture showed upregulated expression (figure 3).

Metabolomic analyses and live cell imaging demonstrate hPCLS viability over time in ex vivo culture To investigate whether hPCLS derived from tissue resected from non-ILD patients are metabolically active in ex vivo culture conditions, samples were harvested as for the proteomic analysis, then solubilised and mechanically homogenised to extract metabolites. Ex vivo cultured hPCLS (three donors, two replicates per donor, two hPCLS per replicate) were subjected to LC/MS-based untargeted metabolomic analysis [32]. A total of 3523 metabolites were detected reproducibly (supplementary table S2) over the range of samples analysed. Out of these, 335 metabolites changed significantly in their abundance from day 1 to 13 (supplementary table S2a; supplementary figure S2e).

Pathway analysis of the significantly changing annotated metabolites revealed no apparent enrichment of specific pathways. We analysed changes in selected metabolites sensitive to alterations in tissue health. 


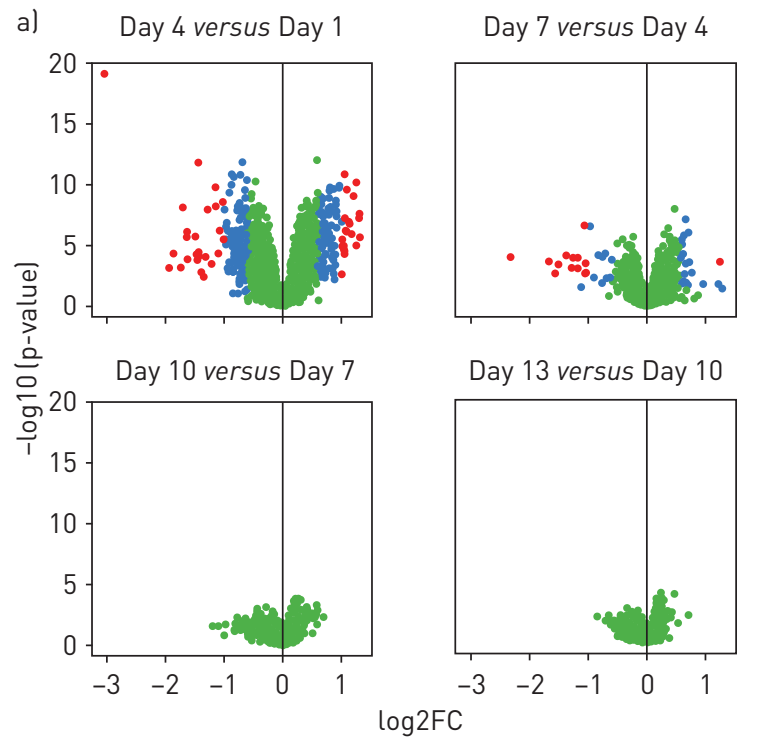

Log2FC $\geqslant 1$ or $\leqslant 1$ and adjusted $p$-value $\leqslant 0.05$

Log $2 F C \geqslant 0.58$ or $\leqslant 0.58$ and adjusted $p$-value $\leqslant 0.05$

Adjusted $p$-value $>0.05$ b)

Day 4 versus Day 1

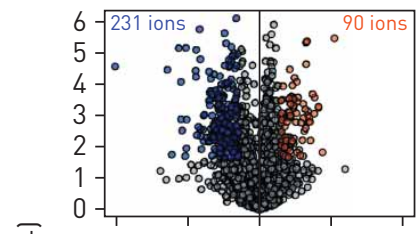

Day 10 versus Day 7

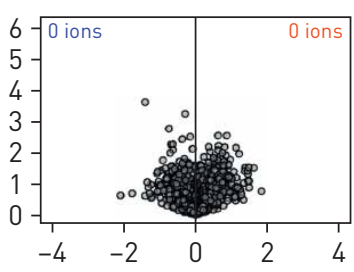

Day 13 versus Day 10

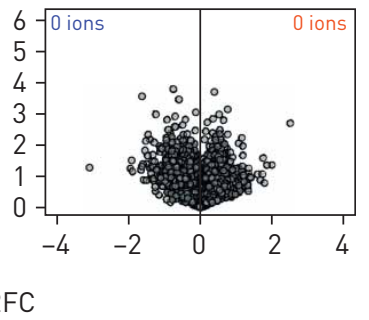

Log2FC $\leqslant 0.58$ and adjusted $p$-value $\leqslant 0.05$

Log2FC $\geqslant 0.58$ and adjusted $p$-value $\leqslant 0.05$ $\mathrm{FDR}>0.1$

FIGURE 2 Multiomic analysis of molecular changes in ex vivo cultured human precision-cut lung slices (hPCLS). hPCLS derived from tissue resections from non-interstitial lung disease (ILD) patients (four donors) in ex vivo culture were harvested on days $1,4,7,10$ and 13 without any treatment and were subjected to mass spectrometry analysis. a) Volcano plot analysis of the proteome of hPCLS cultured ex vivo over time. Log2 fold change (Log2FC) of the respective proteome on a given day of culture was normalised to the previous time point. The analysis highlights that hPCLS undergo moderate changes in the first few days of culture and remain stable over time. b) Untargeted metabolomic analysis of hPCLS was performed. Volcano plot analysis of log2FC changes in ions was performed similarly as above. The analysis confirms that hPCLS undergo very moderate metabolic changes similar to the proteome changes. Donor identification numbers: LT21, LT22, LT24, LT25. Two replicates per donor, two hPCLS per replicate. Supplementary table S6 provides a donor-centric breakdown of mass spectrometry data. FDR: false discovery rate.

This showed that hPCLS are metabolically stable in ex vivo culture (supplementary figure S2f). Specifically, ATP levels of the hPCLS remained unchanged, and L-Lactic acid levels significantly built up over time in culture, implicating a metabolically active 3D tissue system. L-Glutamine was increasingly being metabolised in hPCLS without undergoing any oxidative stress, as depicted by stable levels of the redox-sensitive metabolites cysteine-glutathione disulfide and glutathione (supplementary figure S2f).

To further assess tissue health in hPCLS ex vivo culture, we performed live/dead cell microscopy-based analysis. This showed that there was no change in the signal related to the number of living cells (calcein-AM) from day 1 to day 13 (supplementary figure S2a), while the number of dead cells considerably decreased from day 1 to day 4 by 58\% but remained constant from day 4 to day 13 . The number of dead cells decreased from day 1 to day 7, and remained constant between day 7 and day 13, suggesting that there are always cells dying in the hPCLS culture. By contrast, the calcein-AM signal did not change over time, suggesting active cell proliferation was taking place to replace dying cells (supplementary figure S2a). This is further supported by the constant ATP metabolite levels over time (supplementary figure S2f, g). Consistent with these data, proteomic analysis comparing day 13 to day 1 (supplementary figure S2b) showed that pathways inhibiting cell proliferation were downregulated.

\section{Induction of pro-fibrotic signalling upon TGF- $\beta 1$ stimulation in ex vivo hPCLS culture}

TGF- $\beta 1$ is a cytokine regarded as a master regulator of fibrotic signalling [46]. To induce pro-fibrotic signalling in our culture system, we used TGF- $\beta 1\left(10 \mathrm{ng} \cdot \mathrm{mL}^{-1}\right)$ as reported previously by other groups both in murine model systems [47] and hPCLS [11]. On days 1, 4, 7 and 10 (figure 1), hPCLS derived from tissue resected from non-ILD patients were replenished with fresh medium containing human recombinant TGF- $\beta 1$ or vehicle in ex vivo culture. Supernatants from each hPCLS $( \pm$ TGF- $\beta 1)$ in culture were collected separately on days 4, 7 and 13. Per donor, four hPCLS-conditioned supernatants (except for LT18, day 4) were separately subjected to proprietary competitive ELISA to detect selected neoepitope [48] pro-fibrotic markers such as PRO-C1 [26], FBN-C and C3M. Consistent with earlier studies [11, 49], 


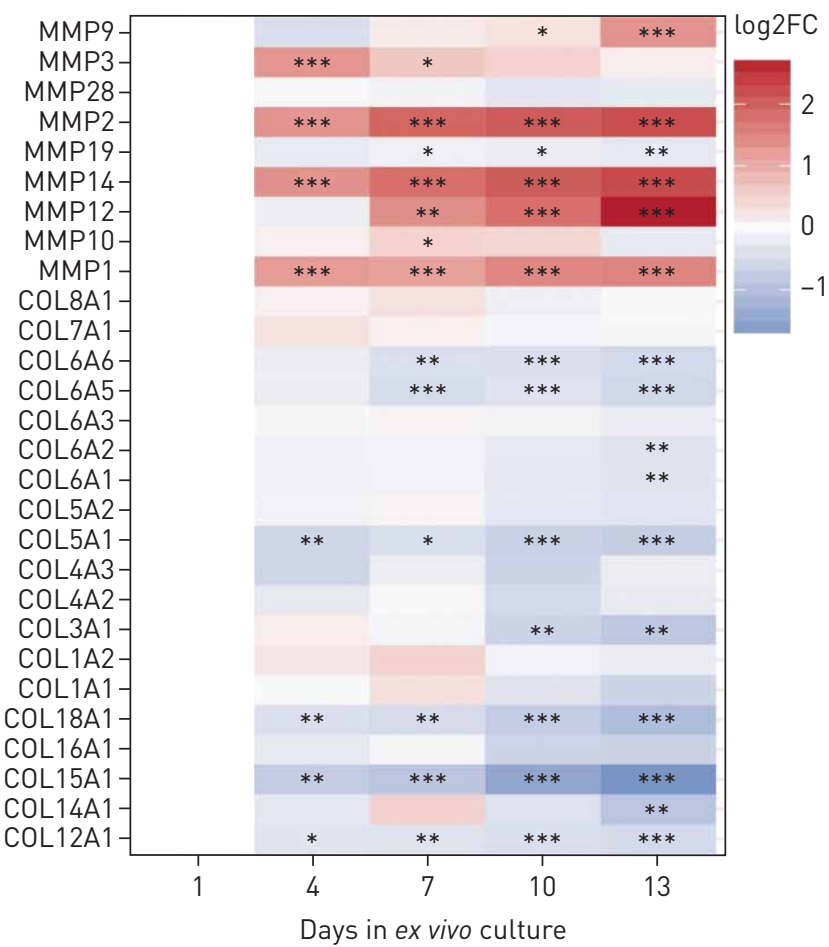

FIGURE 3 Differential regulation of matrix metalloproteinases (MMPs) and collagens (COLs) upon ex vivo culture of human precision-cut lung slices (hPCLS). The heat map shows the log2 fold changes (Log2FC) in the levels of MMPs and different COLs detected in the proteomics analysis of unstimulated hPCLS over time in ex vivo culture. The data show there was consistent upregulation of extracellular matrix degrading and remodelling signalling network proteins with reduced levels of COLs. The Log2FC are calculated with respect to day 1. $p$-values were calculated for the false discovery rates (FDRs). ${ }^{*}: p<0.05 ;{ }^{* \star}: p<0.01 ;{ }^{* \star *}: p<0.001$.

TGF- $\beta 1$ stimulation (figure 4a) increased the level of PRO-C1 and FBN-C detected in the culture supernatants, indicating induction of pro-fibrotic signalling, while C3M showed no changes.

To better characterise how the hPCLS model system responded to TGF- $\beta 1$ stimulation, we also analysed the TGF- $\beta 1$ response at the proteomic level (four donors, two replicates per donor, two hPCLS per replicate). Here, hPCLS (vehicle-treated and TGF- $\beta 1$ treated) were harvested on day 13 of ex vivo culture. The hPCLS were subjected to $2 \%$ SDS solubilisation and mechanical disruption. Subsequently, lysates were subjected to LC/MS. On average, a total of 6600 proteins per replicate and per donor were detected.

Among these, 3998 proteins were common across eight replicates (four donors; supplementary table S4) and were subjected to further downstream analyses. Differential analysis of TGF- $\beta 1$-treated hPCLS compared to vehicle-treated hPCLS (day 13) showed that TGF- $\beta 1$ treatment resulted in a significant change of 109 proteins (Log2FC between 0.58 and -0.58 , FDR p-value of 0.05 , out of 3998 proteins, supplementary figure S4a). A curated data analysis for proteins associated with fibrotic processes showed that proteins such as COL1A2, COL3A1, COL5A1, FBN-C, thrombospondin 1 (THBS1) [50], THBS2 [51], versican (VCAN) [52], TNC [52] and FKBP prolyl isomerase 10 (FKBP10) [53] were significantly upregulated (figure 4b). Furthermore, significantly changing proteins were subjected to pathway analysis using Cytoscape (supplementary figure S4b). This showed induction of pro-fibrotic pathways such as embryonic morphogenesis, ECM organisation and interleukin signalling, and downregulation of pro-epithelial injury signalling pathways such as surfactant metabolism [54-57]. Proteomic analysis of peripheral blood from IPF patients has previously been shown to have downregulated cell proliferation pathways [58], similar to the proteome of TGF- $\beta 1$-treated hPCLS analysed in our study.

The 109 proteins significantly regulated (supplementary figure S4a) upon TGF- $\beta 1$ treatment were curated against the LGEA database for cell type-specific markers (supplementary table S5). This analysis showed that the majority of the significantly downregulated proteins (39 out of 44) are specific for epithelial cells 

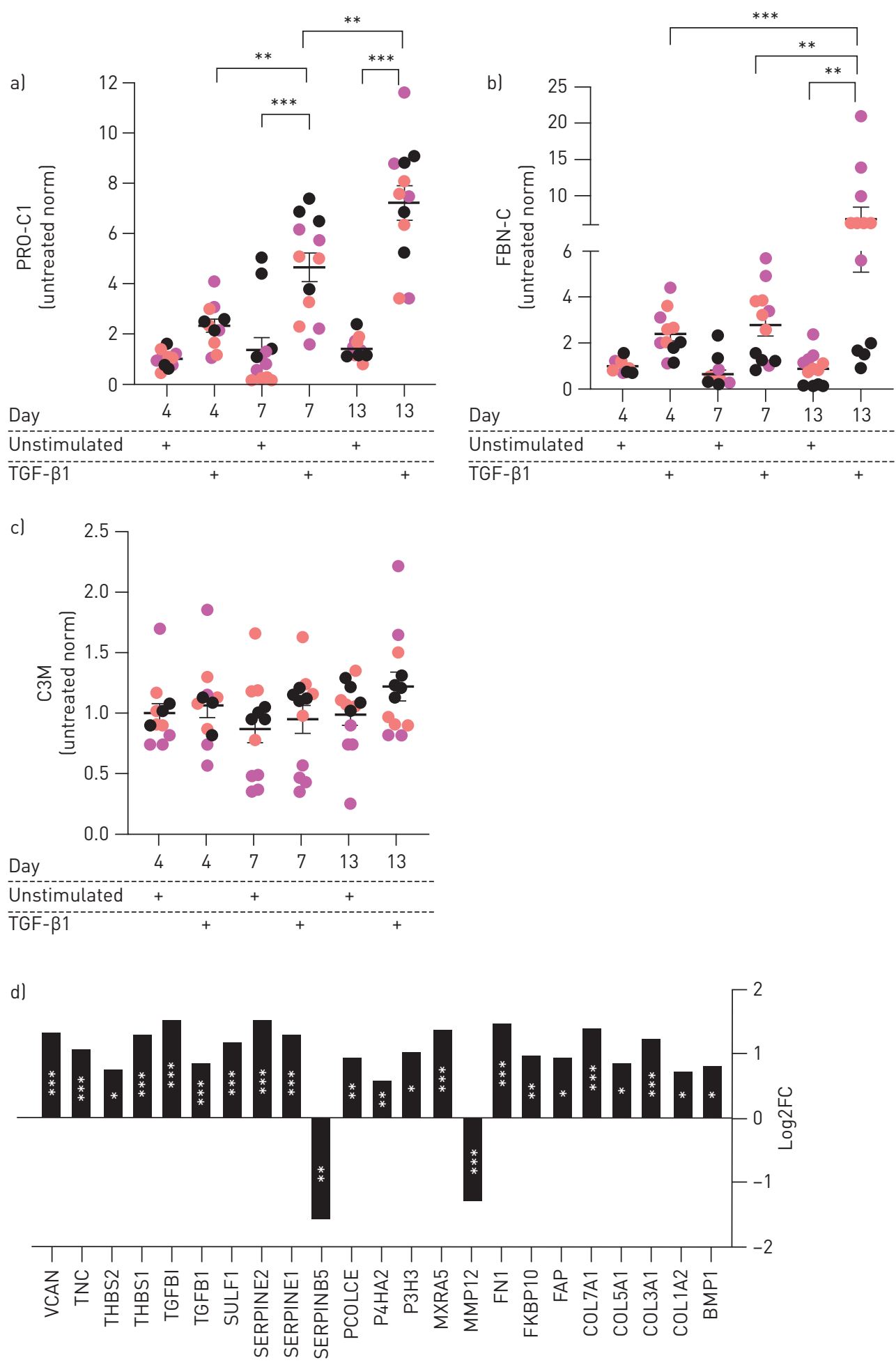

Pro-fibrotic proteins

FIGURE 4 Assessment of pro-fibrotic signalling induction upon transforming growth factor- $\beta 1$ (TGF- $\beta 1$ ) stimulation of ex vivo cultured human precision-cut lung slices (hPCLS) derived from tissue resected from non-interstitial lung disease patients. a-c) ELISA analysis of media supernatants of vehicle-treated (unstimulated) and TGF- $\beta 1$-treated hPCLS (four hPCLS per donor) for procollagen $1 \mathrm{~N}$-terminal propeptide (PRO-C1) (a), C-terminal part of fibronectin (FBN-C) (b) and collagen type III degradation marker (C3M) (c) soluble pro-fibrotic markers. Y-axis "untreated norm" is the absolute amount of these neoepitopes normalised to untreated supernatant in the respective donor. Different colours represent individual donors, and each dot represents a hPCLS. Media supernatant was collected from each hPCLS over time. Absolute concentration (in 
$\mathrm{ng} \cdot \mathrm{mL}^{-1}$ ) was determined (supplementary table S8). All values were normalised to the mean vehicle-treated control (of each donor) on day 4. Results show clear upregulation of the majority of these markers. ELISA data were statistically analysed using a simple one-way ANOVA with Tukey's multiple comparisons test. A ShapiroWilk normality test confirmed data normality. Donor identification numbers: LT08, LT13, LT18. p-values were calculated for the false discovery rates. ${ }^{*}: p<0.05 ;{ }^{\star \star}: p<0.01 ;{ }^{* \star *}: p<0.001$. d) TGF- $\beta 1$ and vehicle-treated hPCLS were harvested on day 13 of ex vivo culture. Data show log2 fold change (Log2FC) of proteins involved in fibrotic signalling upon TGF- $\beta 1$ stimulation on day 13 (with respect to vehicle day 13 ) of hPCLS culture. Donor identification numbers: LT21, LT22, LT24, LT25. Two replicates per donor, two hPCLS per replicate. *: $\log 10$ adjusted $p$-value $<0.05 ;{ }^{* *}$ : $\log 10$ adjusted $p$-value $<0.005 ;{ }^{* * *}$ : $\log 10$ adjusted $p$-value $<0.0005$. Supplementary table $\mathrm{S} 7$ provides a donor-centric breakdown of mass spectrometry data.

while those upregulated (64 out of 65) are specific for mesenchymal cells, suggesting that TGF- $\beta 1$ stimulation might induce an EMT-based pro-fibrotic signalling system [5] under the culture conditions used here. To confirm that it is EMT requires further analyses. Both ELISAs for culture supernatant and the proteomic analyses of the cellular fraction showed high upregulation of collagens upon TGF- $\beta 1$ stimulation (figure 4a, b). In our proteomics data, the typical myofibroblast marker ACTA2 could not be detected, but other markers such as myosin light chain kinase (MYLK) [40] and transgelin (TAGLN) [40] did show significant upregulation (Log2FC 0.52 and 0.69, respectively; supplementary table S4). These data are consistent with several recent studies $[11,19,20]$ showing induction of pro-fibrotic signalling in ex vivo cultured hPCLS using TGF- $\beta 1$ stimulation [11] or a cocktail of pro-fibrotic factors [19].

Whether such upregulation of pro-fibrotic factors with differential regulation of MMPs results in a consistent and specific ECM deposition (fibrillar collagen in particular) has so far not been tested. Therefore, we investigated whether the observed upregulation of pro-fibrotic factors was translated into deposition of fibrillar collagens in the ECM.

\section{Quantitative label-free SHG imaging analysis of fibrillar collagen}

SHG microscopy is a label-free imaging method in which individual non-centrosymmetric molecules generate a second harmonic signal when illuminated with short far-red laser pulses as they are used in two-photon microscopy [59]. In human tissues, the acto-myosin complex of skeletal muscles (cytosolic) [60], large microtubules [61] (cytosolic) and fibrillar collagens [59] (ECM) have been demonstrated to have the non-centrosymmetry required to generate SHG signals. Given the earlier findings that only large fibrillar collagen is present in the ECM of lung tissue [23], SHG imaging can be considered to be a highly specific and suitable technique for quantitative measurements of matrix deposition in hPCLS.

First, we compared hPCLS (PFA fixed on day 0) derived from tumour-free lung tissue of non-ILD patients with those derived from end-stage ILD (IPF and nonspecific interstitial pneumonia) patients. $250 \mu \mathrm{m}$ thick and $8 \mathrm{~mm}$ diameter hPCLS from day 0 were chemically fixed. The entire hPCLS was imaged (figure $5 a-c$ ). Subsequently, the acquired 3D stacks were analysed using a semi-automated image analysis pipeline (Jython script-image analysis, supplementary material). The raw SHG intensity values (supplementary figure S5a) of non-ILD and ILD patients were significantly different. To quantify these relative fold differences, the integrated SHG signal (SHGi) was log2 transformed (Log2SHGi) for each hPCLS, and the average of SHGi of all non-ILD hPCLS (four non-ILD patients) was calculated and log2 transformed (Log2SHGic). Next, for each hPCLS the difference between Log2SHGi and Log2SHGic was determined as Log2SHGi healthy-norm (normalised to healthy donors). Here, the number of ILD hPCLS was normalised to the total of non-ILD controls as per the logarithmic formula, $\log (\mathrm{m} / \mathrm{n})=\log (\mathrm{m})-\log (\mathrm{n})$. The analysis showed significantly more deposited fibrillar collagen in ILD hPCLS than in non-ILD controls. We observed that the SHG signal originating from airways or blood vessels (figure 5b) was similar in intensity between non-ILD and ILD tissues, while the interstitial collagen signal (figure 5b) showed a dramatic increase in ILD compared to non-ILD conditions.

\section{hPCLS have variable quantitative SHG signal due to spatial morphological differences}

Visual analysis of SHG images of whole hPCLS (PFA fixed, unstimulated, non-ILD patients, day 13) showed inherent heterogeneity of the SHG signal (figure 5d). Clearly, different hPCLS have varied content of airways, blood vessels and lung interstitium. Therefore, based on our observation (figure 5b) and previous reports that it is the parenchymal interstitium that is affected [62] in fibrotic phenotypes in ILDs, we devised a two-way image analysis approach: 1) SHG intensity from whole hPCLS was analysed to compare differences and 2) any ROI that had an SHG signal originating from a blood vessel or an airway or had no signal was manually excluded from the analysis (figure 5e). Hence, only the interstitium of the 
a)

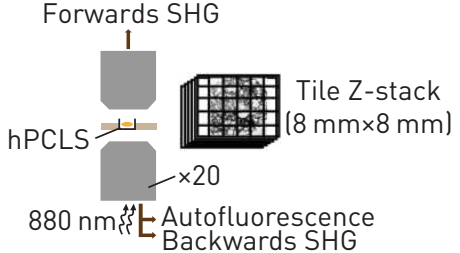

c)

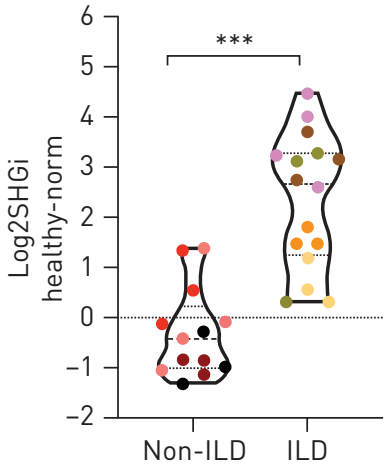

e)

FIJI semi-automated script workflow

a) Threshold

b) Division into ROI tiles $0.8 \mathrm{~mm} \times 0.8 \mathrm{~mm}$

c) Only 3D ROIs of alveoli and interstitium quantified (manual selection)

d) 3D integrated density/ROI

e) Total fibrillar collagen $=F S H G_{\text {intensity }}+\mathrm{BSHG}_{\text {intensity }}$

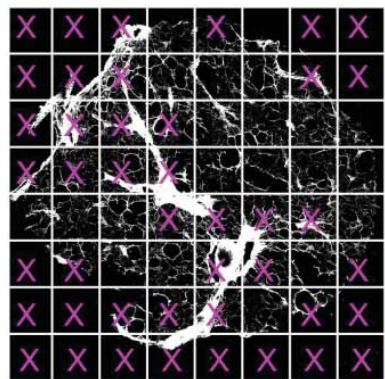

b)
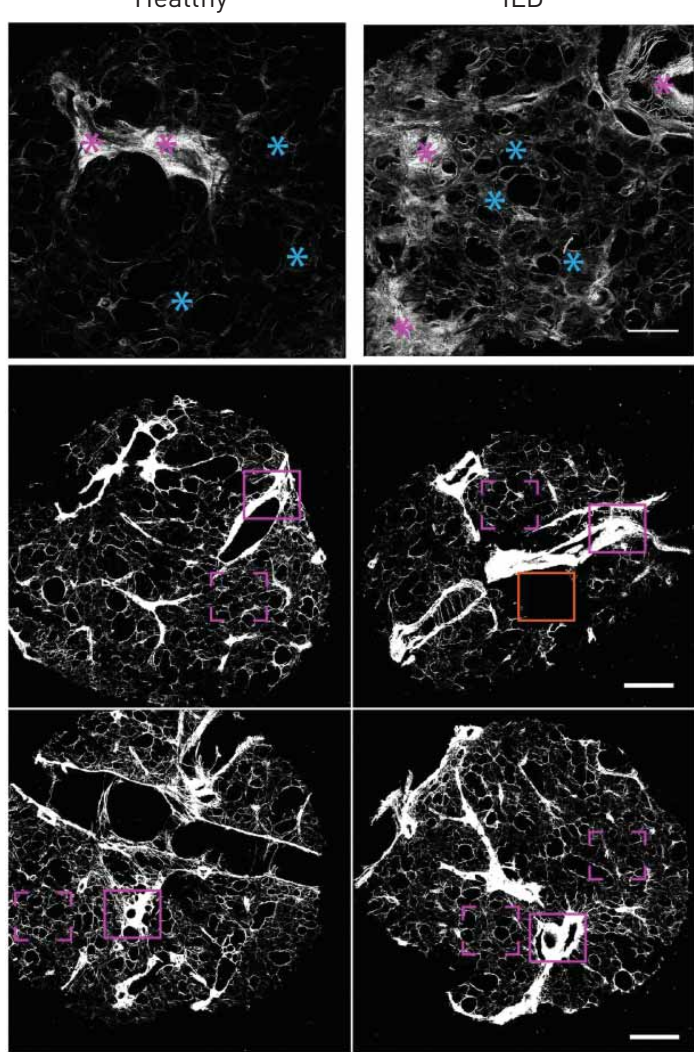

\ulcorner\urcorner Interstitial fibrillar collagen SHG signal

Airway/blood vessel fibrillar collagen SHG signal

hPCLS preparation induced damage/artefact f)

FIGURE 5 Second harmonic generation (SHG) image analysis of fibrillar collagen in human precision-cut lung slices (hPCLS). a) Scheme representing the SHG microscopy and imaging set-up. hPCLS were imaged in a 3D-tiled scan with a $\times 20$ air objective. b, c) Maximum Z-projection and quantification of SHG z-stacks of paraformaldehyde (PFA)-fixed (day 0) hPCLS from non-interstitial lung disease (non-ILD) and ILD patients. Violin plots show Log2SHGi-normalised values for whole hPCLS. A two-way ANOVA was performed, with disease type and donor to donor variation as factors. ${ }^{\star \star \star}: p<0.001$. Different colours represent individual donors. Four non-ILD donor-derived hPCLS (3-4 hPCLS per donor) and five ILD donor-derived hPCLS (3-4 hPCLS per donor) were used. Donor identification numbers: non-ILD patients: LT04, LT05, LT06, LT07; ILD patients: L340, L348, L323, L324, L315. d) SHG images of hPCLS from non-ILD donor tissue resections that were cultured for 13 days and PFA fixed. Donor identification numbers: LT08, LT13, LT18, LT19, LT35, LT36, LT37, LT38, LT39, LT40, LT44, LT139, ET01, ET02, ET03, ET04, ET05, ET06. e) Workflow for quantifying SHG signal originating from lung interstitium. Semi-automated Fiji-based script divides the SHG 3D image stack of hPCLS into $8 \times 8$ grids $(\sim 0.8 \times 0.8 \mathrm{~mm})$. Magenta " $X$ " represents the example regions of interest (ROIs) that were excluded from the 
analysis. Forwards SHG (FSHG) and backwards SHG (BSHG) channel intensity is added and total fibrillar collagen is calculated. f) Colour of the points in the plots represents hPCLS per donor. Graph shows that whole hPCLS SHG intensity values are more variable than the interstitial ROIs from the same donor pool. Scale bars: $1000 \mu \mathrm{m}$.

hPCLS was analysed to compare differences (figure 5e). To quantitatively measure the differences in the two approaches, we used SHG images of unstimulated hPCLS, PFA fixed on day 13 of ex vivo culture. Using our image analysis pipeline, SHG intensity of whole hPCLS (whole) and just the interstitium (ROIs) was calculated (raw values whole hPCLS supplementary figure S5b). To analyse fold differences, the raw values of SHG intensity (SHGi) of whole hPCLS or just the interstitial ROI values were log2 transformed (Log2SHGi) and normalised to the mean SHG intensity of unstimulated hPCLS (Log2SHGic) of each donor (Log2SHGi unstimulated-donor-norm). To analyse if the presence of heterogeneous fibrillar collagen (originating from airways or blood vessels) makes the SHG signal more variable, we analysed the SHG intensity levels measured by whole hPCLS and the ROI method (figure $5 \mathrm{f}$ ). The whole hPCLS variance (0.39) was two times higher than that of the ROI analysis (0.19). Furthermore, only Log2SHGi values of fibrillar collagen from ROI analysis showed normal distribution as per our normality tests (table 2, Anderson-Darling test, D’Agostino-Pearson test and Shapiro-Wilk test). The data show that ROI analysis removes the inherent variability in hPCLS that comes from factors not relevant to the current analysis. These data suggest that if there are subtle changes taking place in the interstitial fibrillar collagen, ROI analysis will be better suited to quantify these changes.

We also analysed if there were sex, smoking status or age-related difference in interstitial collagen in unstimulated hPCLS after 13 days of ex vivo culture (supplementary figure S5c). The analyses showed that the average fibrillar collagen content was lower in male versus female donors. The average fibrillar collagen content in donors aged $>65$ years was higher than that of those $<65$ years. However, neither difference was statistically significant. Ex-smokers had significantly more fibrillar collagen content than current smokers (supplementary figure S5c). The underlying mechanisms for these differences remain elusive.

\section{hPCLS show variable fibrillar collagen deposition response upon TGF- $\beta 1$ stimulation}

hPCLS derived from the lung parenchyma of non-ILD patients were stimulated with TGF- $\beta 1$ and culture supernatants were harvested on day 13 of ex vivo culture. The supernatants were subjected to ELISA for PRO-C1 and FBN-C (figure 6a, b). The data show an unequivocal upregulated synthesis of both markers upon TGF- $\beta 1$ stimulation across all the donors $(n=10)$. To analyse if the upregulated synthesis of ECM proteins (figure 4b) and PRO-C1 and FBN-C (figure 6a, b) results in a corresponding increase in ECM deposition, we employed our label-free SHG imaging and image analysis pipeline to quantitatively

TABLE 2 Log normality test values for SHG intensity comparison of untreated hPCLS (day 13)

Whole hPCLS

ROI hPCLS

\begin{tabular}{lcc}
\hline Anderson-Darling test & & \\
A2 & 0.9273 & 0.3467 \\
p-value & 0.0176 & 0.4716 \\
Passed normality test $(\alpha=0.1)$ ? & No & Yes \\
p-value summary & $\star$ & NS \\
D'Agostino-Pearson test & & \\
K2 & 5.067 & 1.434 \\
p-value & 0.0794 & 0.4882 \\
Passed normality test $(\alpha=0.1)$ ? & No & Yes \\
p-value summary & NS & NS \\
Shapiro-Wilk test & & \\
W & 0.9520 & 0.9835 \\
p-value & 0.0080 & 0.4784 \\
Passed normality test $(\alpha=0.1)$ ? & No & Yes \\
p-value summary & $\star \star$ & NS \\
\hline
\end{tabular}

SHG: second harmonic generation; hPCLS: human precision-cut lung slice; ROI: region of interest; NS: nonsignificant. *: $p<0.05 ;{ }^{\star \star}: p<0.01$. 
a)

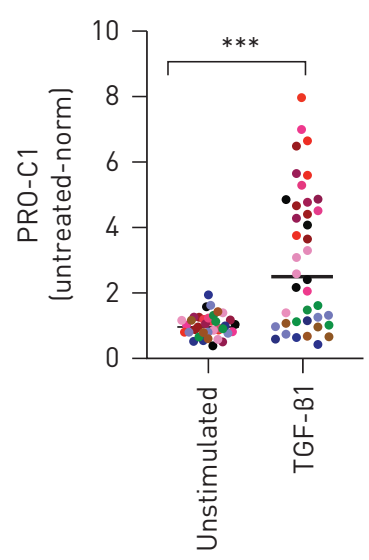

b)

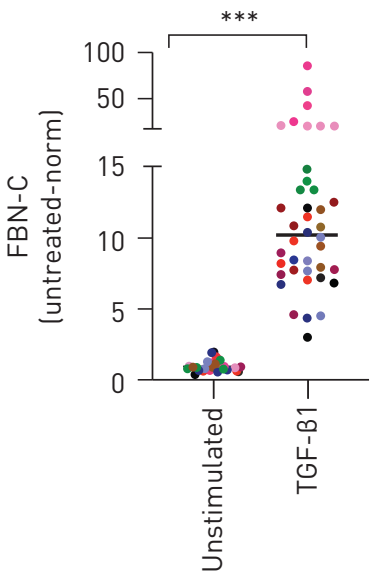

c)

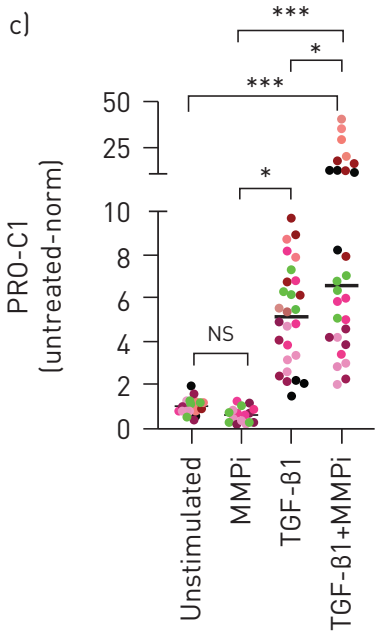

d)

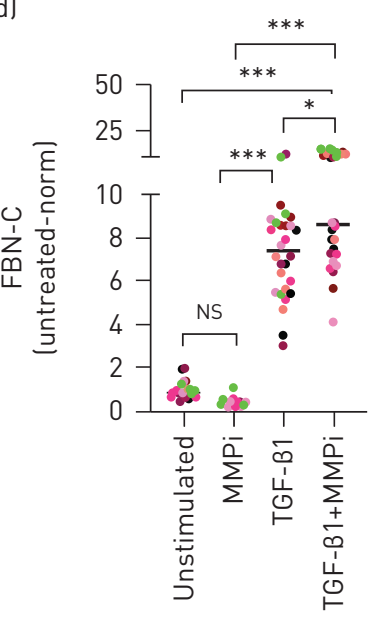

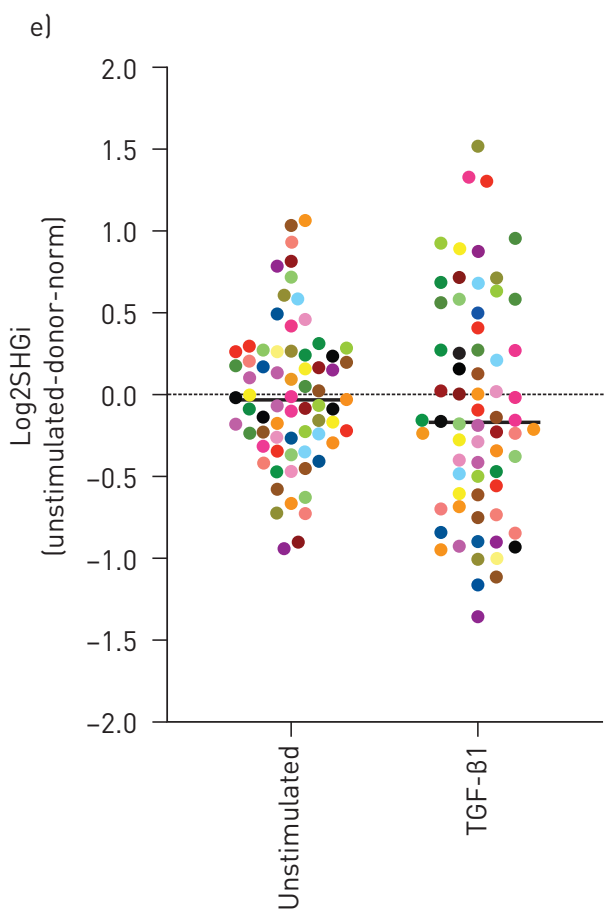

f)

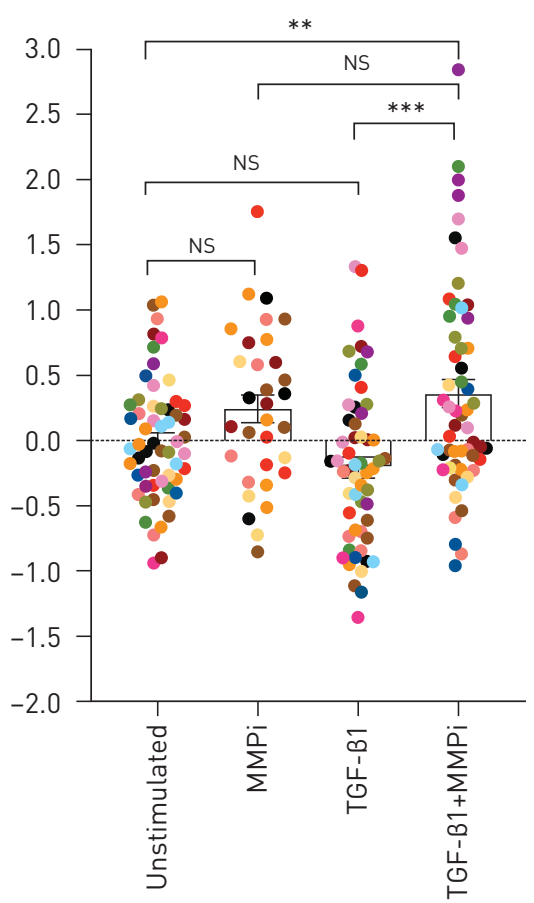

g) 2

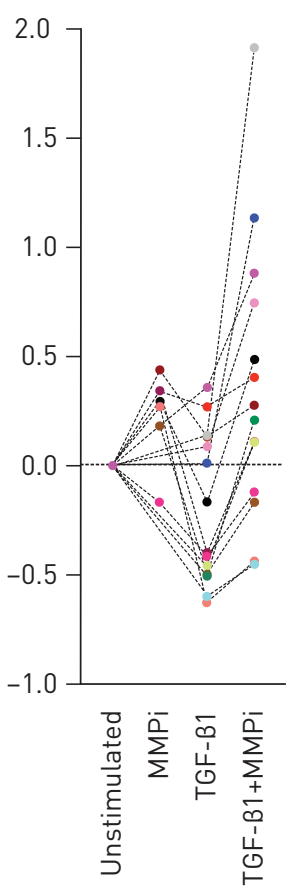

h)

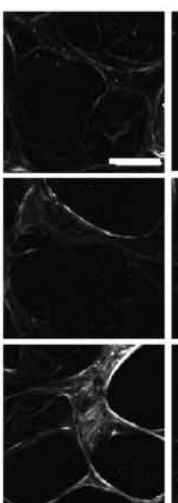

Unstimulated

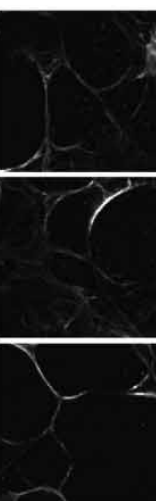

TGF-B1

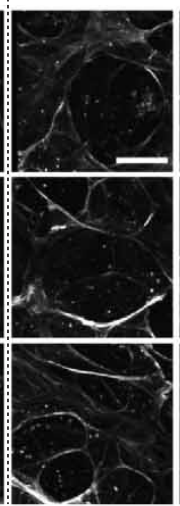

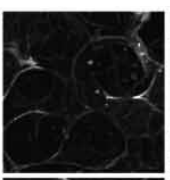

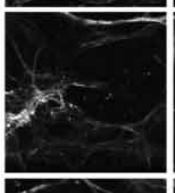

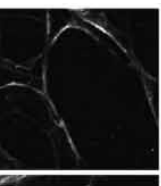
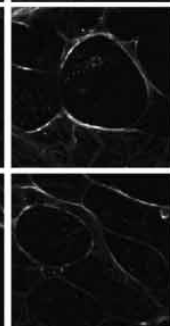
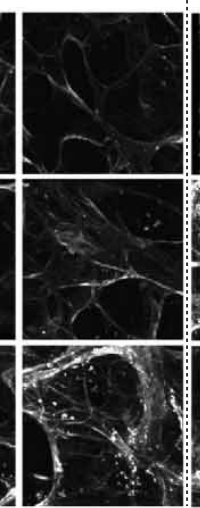

TGF-B1+MMPi

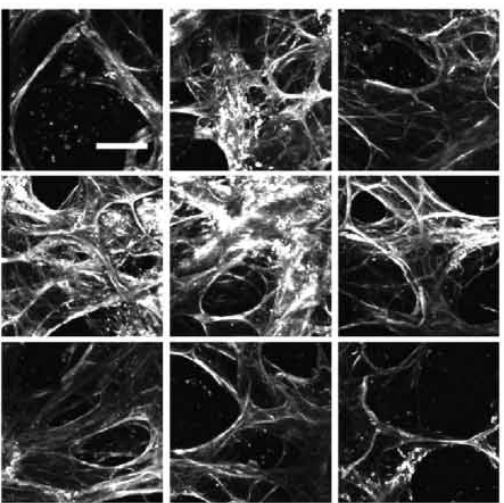


FIGURE 6 Second harmonic generation (SHG) image analysis of fibrillar collagen deposition in ex vivo cultured human precision-cut lung slices (hPCLS) upon stimulation with transforming growth factor- $\beta 1$ (TGF- $\beta 1$ ) and TGF- $\beta 1$ +matrix metalloproteinase inhibitor (MMPi). a, b) Relative amount of procollagen $1 \mathrm{~N}$-terminal propeptide (PRO-C1) and C-terminal part of fibronectin (FBN-C) in the supernatants of vehicle- and TGF- $\beta 1$-stimulated hPCLS. Different colours represent individual donors, and each dot represents a hPCLS. Media supernatant on different days were collected from each hPCLS supernatant. Absolute concentrations $\left(\mathrm{ng} \cdot \mathrm{mL}^{-1}\right.$ ) were determined (supplementary table S9). The results show consistent upregulation of both PRO-C1 and FBN-C upon TGF- $\beta 1$ stimulation across all donors. All values were normalised to the mean vehicle-treated or unstimulated control (of each donor) on day 13 ( $y$-axis, untreated-norm). Donor identification numbers: LT08, LT13, LT18, LT35, LT36, LT37, ET01,

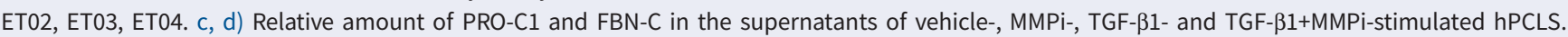
Absolute concentrations ( $\mathrm{ng} \cdot \mathrm{mL}^{-1}$ ) were determined (supplementary table S10). All values were normalised to the mean vehicle-treated control (of each donor) on day 13. Donor identification numbers: LT35, LT36, LT37, ET01, ET02, ET03, ET04. e) The raw values of SHG intensity (iSHG) of interstitial regions of interest (ROIs) from vehicle- and TGF- $\beta 1$-stimulated hPCLS values were log2 transformed (Log2iSHG) and normalised to the mean SHG intensity (Log2iSHGc) of each donor (unlike global normalisation) (3-6 hPCLS per donor, 18 donors). Results (Log2SHGi) show no significant difference in deposited fibrillar collagen upon TGF- $\beta 1$ stimulation. $f$, g) Log2SHGi values of fibrillar collagen in vehicle-, MMPi-, TGF- $\beta 1$ and TGF- $\beta 1+$ MMPi-stimulated hPCLS. g) Mean Log2SHGi of each donor in response to vehicle, MMPi, TGF- $\beta 1$ and TGF- $\beta 1+M M P i$ treatment. Results show a significant increase in fibrillar collagen signal upon TGF- $\beta 1+M M P i$ treatment. Each line connects the response of the same donor. h) Maximum Z-projection of representative ROIs of vehicle-, TGF- $\beta 1$ - and TGF- $\beta 1+M M P i-t r e a t e d ~ h P C L S$ (same donor). Scale bars: $250 \mu \mathrm{m}$. For (a-f), two-way ANOVA (with treatment and donor as two variables) with Tukey's multiple comparisons test was performed. ${ }^{\star}: p<0.05 ;{ }^{\star \star}: p<0.01 ;{ }^{\star \star \star}$ : $p<0.001$. A Shapiro-Wilk test confirmed data normality. For vehicle-, TGF- $\beta 1$ - and TGF- $\beta 1+M M P i-s t i m u l a t e d ~ h P C L S, ~ 14$ donors were analysed with $3-$ 6 hPCLS per donor. For MMPi-stimulated hPCLS, seven donors were analysed. Donor identification numbers: LT08, LT13, LT18, LT19, LT35, LT36, LT37, LT38, LT39, LT40, LT44, ET01, ET02, ET03, ET04, ET05, ET06. The ELISA data from donors LT08, LT13, LT18, LT35, LT36, LT37 on day 13 are the same in figures $4 a$ and $6 c, d$. The data are reused in this figure to represent unequivocal upregulation of PRO-C1 and FBN-C across all donors. NS: nonsignificant.

measure the deposition of fibrillar collagen in unstimulated and TGF- $\beta 1$-stimulated hPCLS (derived from tissue resected from non-ILD patients). The raw values of iSHG of interstitial ROIs from vehicle and TGF- $\beta 1$-stimulated hPCLS values were log2 transformed (Log2iSHG) and normalised to the mean SHGi (Log2iSHGc) of each donor (3-6 hPCLS per donor, 18 donors). In contrast to the consistently elevated levels of PRO-C1 and FBN-C as determined by ELISA measurements, Log2SHGi values of fibrillar collagen showed no significant additional deposition of fibrillar collagen (figure 6e) in the interstitium of hPCLS stimulated with TGF- $\beta 1$ in comparison to control tissue (figure 6h, supplementary figure S5d). Differential proteomic analysis of vehicle and TGF- $\beta 1$-stimulated hPCLS (day 13, supplementary table S4) showed that some of the metalloproteinases specific for degradation of native collagens (type I, II, III, IV, V, VII, X and XI) [45], also known as collagenases, such as MMP2 (Log2FC 0.68), MMP3 (Log2FC 0.65 ) and MMP14 (Log2FC 0.52), showed upregulation in our ex vivo culture system after TGF- $\beta 1$ treatment, while MMP12 (Log2FC -0.97) and MMP9 (Log2FC -1.2) were downregulated. These data are consistent with recent data in murine models of PCLS, in which collagen type I and III and elastin had high turnover due to higher MMP activity [63]. Also, Han et al. [64] have shown that pan inhibition of MMP activity using Ilomastat (GM6001) enhances collagen fibril formation in human mesenchymal stem cells in in vitro cultures.

MMPi together with TGF- 1 1 stimulation significantly enhances deposition of fibrillar collagen in ECM Given the observed differential upregulation of different MMPs in ex vivo culture of hPCLS (figure 3), as stated above (supplementary table S4), and reports that imbalance of MMP activity is crucial to fibrotic tissues [65], 14 donors were additionally treated with a combination of TGF- $\beta 1$ and GM6001, an MMPi. Out of these 14 donors, media supernatants were collected on day 13 for seven. ELISA of hPCLS-conditioned supernatants was performed for PRO-C1 and FBN-C (figure 6c, d). The results showed that MMPi treatment alone (four donors) had no effect on the synthesis of these markers. TGF- $\beta 1$ stimulation expectedly resulted in consistent upregulation of both these markers compared to unstimulated hPCLS supernatants. Concomitant stimulation of TGF- $\beta 1$ and MMPi resulted in an increased amount of both PRO-C1 and FBN-C compared to TGF- $\beta 1$ treatment alone (figure 6c, d).

To investigate whether treating hPCLS with TGF- $\beta 1+M M P i$ resulted in increased deposition of fibrillar collagen in lung ECM, we analysed vehicle-, MMPi-, TGF- $\beta 1$ - and TGF- $\beta 1+$ MMPi-stimulated hPCLS using our SHG workflow. MMPi treatment alone had no statistically significant effect on the interstitial collagen SHG signal compared to the respective vehicle-treated hPCLS (figure 6f). Concomitant treatment of TGF- $\beta 1$ and MMPi resulted in significantly more deposition of fibrillar collagen compared to TGF- $\beta 1$ treatment alone (figure 6f). Patient-specific analysis of the data (figure 6g) showed that TGF- $\beta 1$ treatment caused a decrease in some and an increase in others of SHG signal compared to control hPCLS. The reasons for this differential response to TGF- $\beta 1$ treatment are currently unclear. However, for every sample 
analysed, concomitant treatment with MMPi resulted in a consistently higher fibrillar collagen signal compared to TGF- $\beta 1$ treatment alone. Together, these data confirm that metalloproteinase expression/ activity is a rate-limiting factor in the hPCLS model system characterised here for deposition of fibrillar collagen upon TGF- $\beta 1$ stimulation.

\section{Discussion}

hPCLS have emerged as a model system with high potential for recapitulating pathophysiological conditions close to that of human physiology $[11,19]$. However, characterisation of the inherent molecular changes that such a system undergoes in ex vivo culture has not been reported to date.

In this study, we report for the first time proteomic and metabolomic changes of hPCLS over 2 weeks of ex vivo culture. This is significantly longer than the 7 days previously reported $[11,19]$. These cell-type data (supplementary figure S2c) were derived from curation of our phenotypic protein expression data to that of RNA-seq data available in the LGEA database. Comparison of the LGEA database sequencing list and recently published single-cell sequencing results of human lung tissue [66-68] shows that some markers classified in the LGEA database as cell type-specific are actually present in more than one cell subtype (e.g. TAGLN, ACTA2, PDGFRA) [68]. Nonetheless, we show that proteins exclusively expressed in the four major cell types [40] of lung tissue are present after 2 weeks of ex vivo culture (supplementary figure S2c). The data also revealed (figure 3 and supplementary figure S2b) the presence of a persistent ECM degradative inflammatory activity during the 2 weeks of ex vivo culture, which is assumed not to be present under healthy physiological conditions. This finding challenges the general assumption in the field that physiologically healthy conditions are preserved in ex vivo culture of hPCLS [21]. The source of this inflammation activity is currently unclear. One explanation could be that it is due to sample preparation, specifically, tissue slicing. Alternatively, it cannot be excluded that the occurrence of the degradative inflammatory activity results from the fact that the tissue slices used in our study were derived from tumour-adjacent areas, which have previously been shown to be immunologically active [69]. Because these changes occur mainly during the first 4 days in culture, it may be advisable in future studies to start investigations after this "stabilising period". However, our studies show that there is already loss of protein expression in lung cell markers, such as SFTPC and AGER, within the first 4 days of culture. Therefore, depending on the question addressed, we consider it important to compare results obtained in the "stabilising period" with the first 4 days of culture. Furthermore, it would be interesting to test if treating hPCLS with cell culture levels of hydrocortisones might be useful to prevent this inflammatory activity.

The presence of cell types such as epithelial, endothelial and immune cells (supplementary figure S2c) suggests the possibility that these cell types transition into myofibroblasts upon stimulation with cytokines such as TGF- $\beta 1$ [3], thereby eliciting a physiologically relevant pro-fibrotic response. Consistent with other studies [11], our proteomic analyses showed that many pro-fibrotic markers were upregulated after TGF- $\beta 1$ treatment (figure 4a, b) including pro-fibrotic fibrillar collagens COL1, COL3 and COL5. On day 13 of hPCLS ex vivo culture, in total 109 proteins were significantly regulated (both up- and downregulated). Strikingly, 64 out of 65 upregulated proteins were characteristic of mesenchymal cell type, and 40 out of 44 downregulated genes were specific to epithelial cell type (supplementary figure S4c). These data suggest that the pro-fibrotic signal induced after TGF- $\beta 1$ treatment of hPCLS might be due to EMT [3]. Alternatively, the downregulation of epithelial proteins with a concomitant increase in mesenchymal markers could be indicative of epithelial cell death or simply impairment of epithelial genes with a concomitant increase in mesenchymal markers in mesenchymal cells. To test this hypothesis further, complementary analyses such as single-cell RNA-seq could be used. Importantly, these experiments are now possible owing to recent advances in single-cell and high-quality RNA preparation from hPCLS [70]. Furthermore, to visualise the dynamics of single cell types in hPCLS and test if they are consistent with our proteomics data, our live imaging set-up can be coupled to that of AкRAм et al. [21].

Other studies [11, 19] have used hPCLS to study fibrotic signalling; however, a readout that measures ECM deposition during ex vivo culture has been lacking. Similarly, in other model systems, indirect readouts such as ELISA [26] of procollagen peptides or hydroxyproline levels in serum have been used to infer fibrosis, e.g. in liver, kidney or lung [71-73]. In the hPCLS model system, collagen has been qualitatively monitored using antibodies and immunohistochemistry approaches [19]. However, the antibodies used in these studies could not discriminate between fibrillar and non-fibrillar collagen types. In order to overcome these limitations, we applied label-free SHG imaging to specifically quantify the fibrillar collagen content present in the ECM of hPCLS. A key advantage of label-free imaging is its ability to image deep into the tissues and specifically measure fibrillar ECM-deposited collagen. SHG imaging analysis of non-ILD and ILD patient-derived hPCLS showed higher levels of fibrillar collagen in ILD-derived hPCLS than in tissues from non-ILD donors (figure 5b, c), showing the quantitative nature of 
the imaging workflow established here. Our image analysis shows that documenting interstitial collagen levels as a function of age, sex and smoking status (supplementary figure S5c) together with other lung vitals could prove to be a powerful tool to detect susceptibility to ILD in humans. This can be combined with other molecular techniques to pin down underlying causes, giving novel insights into the disease mechanism. Interestingly, the increased expression of pro-fibrotic markers in TGF- $\beta 1$-stimulated hPCLS, as revealed by our ELISA and proteomics analyses (PRO-C1 and COL1A2, respectively), did not result in a corresponding significant increase of deposition in ECM (figure 6e-g). This observation could possibly be explained by differential regulation of MMPs and their respective enzymatic activities in non-ILD patient-derived hPCLS upon TGF- $\beta 1$ treatment (supplementary table S4). Here, we observed that MMP9 and MMP12 were significantly downregulated; these two MMPs might have an immune response-related role in hPCLS because both these enzymes have been described to regulate shedding of CD14 receptor to influence innate host defence [74]. The only detected TIMP, TIMP3, showed no significant change. MMP2 and MMP14, however, were upregulated (supplementary table S4). The latter have been known to use COL1, COL2 and COL3 as substrates [45]; therefore, in this case, upregulation might have resulted in enhanced COL degradation before deposition [45]. In support of this hypothesis, concomitant treatment with TGF- $\beta 1$ and GM6001, an MMPi, increased the levels of PRO-C1 and FBN-C (figure 6c, d) in conditioned supernatants, and increased fibrillar collagen deposition in comparison to TGF- $\beta 1$ treatment alone. Consistent with existing literature, these data underline the importance of MMP activity for fibrillar collagen deposition and thus the development of fibrosis. Because GM6001 is a broad MMPi, more experiments will be necessary to determine which MMPs in particular participate in this process. It would also be interesting to investigate the effect on fibrillar collagen deposition quantitatively by stimulating hPCLS with cocktails of pro-inflammatory molecules, because the effect has successfully been demonstrated qualitatively by ALsAFADI et al. [19].

Pulmonary fibrosis is essentially an ageing-related disorder [75] that develops over several years. Therefore, increases of fibrillar collagen deposition within 2 weeks can be expected to be only modest, as observed here. However, we show that concomitant treatment with TGF- $\beta 1$ and an MMPi leads to measurably enhanced fibril deposition in response to TGF- $\beta 1$ treatment in this ex vivo model system, demonstrating the potential of our integrated approach. Interestingly, not all patients' samples responded equally to TGF- $\beta 1$ : out of the 18 donors tested, two showed no difference, eight showed an increased signal and eight showed a decreased signal (figure 6e-g, supplementary figure S5d). Dividing the response of the donors according to their sex, smoking status and age (supplementary figure S5d, e) did not reveal any obvious factor that might explain these variations in TGF- $\beta 1$ response. One explanation could be that certain hPCLS are more predisposed for ECM deposition than others owing to their spatial origin in the lung. Tissue spatial variation of developing fibrotic foci has been reported to be a characteristic of pulmonary fibrosis development [76]. We can therefore not exclude the possibility that the variation of SHG signal in response to TGF- $\beta 1$ treatment is caused by such spatial variations. We can also not exclude the possibility that the variability in TGF- $\beta 1$ activity was caused by variations in stiffness $[77,78]$ of the ECM of hPCLS.

Our multiomic characterisation of hPCLS shows that they are metabolically viable and contain all lung-relevant cell types for up to 2 weeks in ex vivo culture. These data support the idea that a hPCLS model system can be used to study human lung physiology-related molecular processes. However, apparent limitations exist. Our proteomic analysis shows that hPCLS in culture undergo significant changes in MMPs, collagens and pro-inflammatory pathways. This is in contrast to what has been proposed or assumed in earlier studies [79] lacking such multiomic analyses. Recent studies show that after the onset of disease, e.g. initial fibroblast activation and ECM accumulation, recruitment of circulating immune cells further enhance fibrosis [80]. In the hPCLS model system, these cells do survive, but their constant replenishment as it occurs in the human body is still missing. Addition of these circulating cells from the same donor to the hPCLS culture at different days would be a powerful tool to establish their role in fibrosis progression. Furthermore, physiological aspects of lung compliance such as cycling oxygen levels and changes in mechanical tension are lacking. A key addition to improve the current experimental culture set-up could therefore be to culture hPCLS in a bioreactor system that mimics these important components of lung physiology.

IPF lungs develop fibrotic foci, rather than diffuse fibrosis, and these have been extensively characterised by Jones et al. [76]. The image analysis approach developed here focuses on interstitium of lung parenchyma and could in the future help to identify the progression and development of such foci in a quantitative manner. We propose that the complementary approach described here, using label-free imaging of hPCLS together with the measurement of soluble markers such as PRO-C1, FBN-C and C3M, could enable the dynamics of collagen synthesis and degradation (the fractional synthesis) to be characterised ex vivo upon fibrosis progression. Furthermore, to dissect the role of single cell types in mediating the effects 
of various pro-fibrotic stimuli, our workflow could be coupled to the newly established single-cell analysis pipeline in hPCLS by STEgmayr et al. [70].

Because SHG does not require sample fixation, as is the case for immunochemistry, this label-free approach should allow time-lapse studies monitoring the kinetics of ECM deposition over extended periods of time. An initial proof of concept experiment has shown the feasibility of this approach (supplementary figure S6). Live SHG imaging will allow the kinetics of fibrillar collagen deposition, as well as regression, to be assessed upon therapeutic or pharmacological intervention.

In summary, our SHG imaging set-up and analysis pipeline established here will be a powerful tool for studying fibrosis in the future.

Acknowledgements: Technical assistance from Christa Stolp from Biomaterial Bank Heidelberg (BMBH) in tissue assembling is gratefully acknowledged. We would also like to acknowledge the help of Vikki Barrett (GSK Stevenage) for help in training with Krumdieck. We sincerely thank Katrin Strohmer and Anna Rutkowska-Klute (Cellzome-GSK) for their help in setting up the SHG imaging. The Pepperkok team and all the EMBL core facilities are also acknowledged for their support, manuscript preparation and fruitful discussions for data analysis.

Conflict of interest: M.M. Khan has nothing to disclose. D. Poeckel has nothing to disclose. A. Halavatyi has nothing to disclose. J. Zukowska-Kasprzyk has nothing to disclose. F. Stein has nothing to disclose. J. Vappiani has nothing to disclose. D.C. Sevin has nothing to disclose. C. Tischer has nothing to disclose. N. Zinn has nothing to disclose. J.D. Eley has nothing to disclose. N.S. Gudmann has nothing to disclose. T. Muley has nothing to disclose. H. Winter has nothing to disclose. A.J. Fisher reports grants from GlaxoSmithKline, during the conduct of the study, and grants from Pfizer, Nuformix and Genentech, outside the submitted work. C.B. Nanthakumar has nothing to disclose. G. Bergamini has nothing to disclose. R. Pepperkok has nothing to disclose.

Support statement: The research was funded by a joint EMBL-GSK postdoctoral programme and by the German Centre for Lung Research (DZL). Funding information for this article has been deposited with the Crossref Funder Registry.

\section{References}

1 Sakai N, Tager AM. Fibrosis of two: epithelial cell-fibroblast interactions in pulmonary fibrosis. Biochim Biophys Acta 2013; 1832: 911-921.

2 Wynn TA. Common and unique mechanisms regulate fibrosis in various fibroproliferative diseases. J Clin Invest 2007; 117: 524-529.

3 Wynn TA. Cellular and molecular mechanisms of fibrosis. J Pathol 2008; 214: 199-210.

4 Interstitial lung diseases. In: Gibson GJ, Loddenkemper R, Sibille Y, et al., eds. European Lung White Book. Sheffield, European Respiratory Society, 2013.

5 Chapman HA. Epithelial-mesenchymal interactions in pulmonary fibrosis. Annu Rev Physiol 2011; 73: 413-435.

6 Pardo A, Selman M. Matrix metalloproteases in aberrant fibrotic tissue remodeling. Proc Am Thorac Soc 2006; 3: 383-388.

7 Herrera J, Forster C, Pengo T, et al. Registration of the extracellular matrix components constituting the fibroblastic focus in idiopathic pulmonary fibrosis. JCI Insight 2019; 4: e125185.

8 Karsdal MA, Nielsen SH, Leeming DJ, et al. The good and the bad collagens of fibrosis - their role in signaling and organ function. Adv Drug Deliv Rev 2017; 121: 43-56.

9 Nanthakumar CB, Hatley RJ, Lemma S, et al. Dissecting fibrosis: therapeutic insights from the small-molecule toolbox. Nat Rev Drug Discov 2015; 14: 693-720.

10 Fregonese L, Eichler I. The future of the development of medicines in idiopathic pulmonary fibrosis. BMC Med 2015; 13: 239.

11 Roach KM, Sutcliffe A, Matthews L, et al. A model of human lung fibrogenesis for the assessment of anti-fibrotic strategies in idiopathic pulmonary fibrosis. Sci Rep 2018; 8: 342.

12 Hancock LA, Hennessy CE, Solomon GM, et al. Muc5b overexpression causes mucociliary dysfunction and enhances lung fibrosis in mice. Nat Commun 2018; 9: 5363.

13 Tashiro J, Rubio GA, Limper AH, et al. Exploring animal models that resemble idiopathic pulmonary fibrosis. Front Med 2017; 4: 118.

14 Sleijfer S. Bleomycin-induced pneumonitis. Chest 2001; 120: 617-624.

15 Selman M. From anti-inflammatory drugs through antifibrotic agents to lung transplantation: a long road of research, clinical attempts, and failures in the treatment of idiopathic pulmonary fibrosis. Chest 2002; 122: 759-761. 
16 Raghu G, Anstrom KJ, King TE Jr, et al. Azathioprine, and N-acetylcysteine for pulmonary fibrosis. $N$ Engl J Med 2012; 366: 1968-1977.

17 Stefaniak MS, Gandolfi AJ, Brendel K, et al. Agar-filled rat lung slices for use in toxicologic evaluations. Toxicologist 1990; 10: 339.

18 Fisher RL, Smith MS, Hasal SJ, et al. The use of human lung slices in toxicology. Hum Exp Toxicol 1994; 13: 466-471.

19 Alsafadi HN, Staab-Weijnitz CA, Lehmann M, et al. An ex vivo model to induce early fibrosis-like changes in human precision-cut lung slices. Am J Physiol Lung Cell Mol Physiol 2017; 312: L896-L902.

20 Lehmann M, Buhl L, Alsafadi HN, et al. Differential effects of nintedanib and pirfenidone on lung alveolar epithelial cell function in ex vivo murine and human lung tissue cultures of pulmonary fibrosis. Respir Res 2018; 19: 175.

21 Akram KM, Yates LL, Mongey R, et al. Live imaging of alveologenesis in precision-cut lung slices reveals dynamic epithelial cell behaviour. Nat Commun 2019; 10: 1178.

22 Organ LA, Duggan AR, Oballa E, et al. Biomarkers of collagen synthesis predict progression in the PROFILE idiopathic pulmonary fibrosis cohort. Respir Res 2019; 20: 148.

23 Cox G, Kable E, Jones A, et al. 3-Dimensional imaging of collagen using second harmonic generation. J Struct Biol 2003; 141: 53-62.

24 Mercer PF, Woodcock HV, Eley JD, et al. Exploration of a potent PI3 kinase/mTOR inhibitor as a novel anti-fibrotic agent in IPF. Thorax 2016; 71: 701-711.

25 Gerckens M, Alsafadi HN, Wagner DE, et al. Generation of human 3D lung tissue cultures (3D-LTCS) for disease modeling. J Vis Exp 2019; 144 [https://doi.org/10.3791/58437].

26 Leeming DJ, Larsen DV, Zhang C, et al. Enzyme-linked immunosorbent serum assays (ELISAs) for rat and human N-terminal pro-peptide of collagen type I (PINP)-assessment of corresponding epitopes. Clin Biochem 2010; 43: 1249-1256.

27 Barascuk N, Veidal SS, Larsen L, et al. A novel assay for extracellular matrix remodeling associated with liver fibrosis: an enzyme-linked immunosorbent assay (ELISA) for a MMP-9 proteolytically revealed neo-epitope of type III collagen. Clin Biochem 2010; 43: 899-904.

28 Bager CL, Gudmann N, Willumsen N, et al. Quantification of fibronectin as a method to assess ex vivo extracellular matrix remodeling. Biochem Biophys Res Commun 2016; 478: 586-591.

29 Bantscheff M, Schirle M, Sweetman G, et al. Quantitative mass spectrometry in proteomics: a critical review. Anal Bioanal Chem 2007; 389: 1017-1031.

30 Werner T, Sweetman G, Savitski MF, et al. Ion coalescence of neutron encoded TMT 10-plex reporter ions. Anal Chem 2014; 86: 3594-3601.

31 Savitski MM, Mathieson T, Zinn N, et al. Measuring and managing ratio compression for accurate iTRAQ/TMT quantification. J Proteome Res 2013; 12: 3586-3598.

32 Fuhrer T, Heer D, Begemann B, et al. High-throughput, accurate mass metabolome profiling of cellular extracts by flow injection-time-of-flight mass spectrometry. Anal Chem 2011; 83: 7074-7080.

33 Wishart DS, Feunang YD, Marcu A, et al. HMDB 4.0: the human metabolome database for 2018. Nucleic Acids Res 2018; 46: D608-D617.

34 Ritchie ME, Phipson B, Wu D, et al. limma powers differential expression analyses for RNA-sequencing and microarray studies. Nucleic Acids Res 2015; 43: e47.

35 Huber W, von Heydebreck A, Sültmann H, et al. Variance stabilization applied to microarray data calibration and to the quantification of differential expression. Bioinformatics 2002; 18: Suppl. 1, S96-S104.

36 Shannon P, Markiel A, Ozier O, et al. Cytoscape: a software environment for integrated models of biomolecular interaction networks. Genome Res 2003; 13: 2498-2504.

37 Schindelin J, Arganda-Carreras I, Frise E, et al. Fiji: an open-source platform for biological-image analysis. Nat Methods 2012; 9: 676-682.

38 Mi H, Muruganujan A, Ebert D, et al. PANTHER version 14: more genomes, a new PANTHER GO-slim and improvements in enrichment analysis tools. Nucleic Acids Res 2019; 47: D419-D426.

39 Du Y, Guo M, Whitsett JA, et al. 'LungGENS': a web-based tool for mapping single-cell gene expression in the developing lung. Thorax 2015; 70: 1092-1094.

40 Du Y, Kitzmiller JA, Sridharan A, et al. Lung Gene Expression Analysis (LGEA): an integrative web portal for comprehensive gene expression data analysis in lung development. Thorax 2017; 72: 481-484.

41 Benjamin MM, Khalil RA. Matrix metalloproteinase inhibitors as investigative tools in the pathogenesis and management of vascular disease. Exp Suppl 2012; 103: 209-279.

42 Brew K, Nagase $\mathrm{H}$. The tissue inhibitors of metalloproteinases (TIMPs): an ancient family with structural and functional diversity. Biochim Biophys Acta 2010; 1803: 55-71.

43 Lu KV, Jong KA, Rajasekaran AK, et al. Upregulation of tissue inhibitor of metalloproteinases (TIMP)-2 promotes matrix metalloproteinase (MMP)-2 activation and cell invasion in a human glioblastoma cell line. Lab Invest 2004; 84: 8-20. 
44 Wang Z, Juttermann R, Soloway PD. TIMP-2 is required for efficient activation of proMMP-2 in vivo. J Biol Chem 2009; 275: 26411-26415.

45 Chelladurai P, Seeger W, Pullamsetti SS. Matrix metalloproteinases and their inhibitors in pulmonary hypertension. Eur Respir J 2012; 40: 766-782.

46 Meng X, Nikolic-paterson DJ, Lan HY. TGF- $\beta$ : the master regulator of fibrosis. Nat Rev Nephrol 2016; 12 : 325-338.

47 Oenema TA, Maarsingh H, Smit M, et al. Bronchoconstriction induces TGF- $\beta$ release and airway remodelling in guinea pig lung slices. PLoS One 2013; 8: e65580.

48 Leeming DJ, Sand JM, Nielsen MJ, et al. Serological investigation of the collagen degradation profile of patients with chronic obstructive pulmonary disease or idiopathic pulmonary fibrosis. Biomark Insights 2012; 7: 119-126.

49 Huang X, Li L, Ammar R, et al. Molecular characterization of a precision-cut rat lung slice model for the evaluation of antifibrotic drugs. Am J Physiol Lung Cell Mol Physiol 2019; 316: 348-357.

50 Sweetwyne MT, Murphy-Ullrich JE. Thrombospondin1 in tissue repair and fibrosis: TGF- $\beta$-dependent and independent mechanisms. Matrix Biol 2012; 31: 178-186.

51 Reinecke $\mathrm{H}$, Robey TE, Mignone JL, et al. Lack of thrombospondin-2 reduces fibrosis and increases vascularity around cardiac cell grafts. Cardiovasc Pathol 2013; 22: 91-95.

52 Estany S, Vicens-Zygmunt V, Llatjós R, et al. Lung fibrotic tenascin-C upregulation is associated with other extracellular matrix proteins and induced by TGF $\beta 1$. BMC Pulm Med 2014; 14: 120.

53 Staab-Weijnitz CA, Fernandez IE, Knüppel L, et al. FK506-binding protein 10, a potential novel drug target for idiopathic pulmonary fibrosis. Am J Respir Crit Care Med 2015; 192: 455-467.

54 Fernandez IE, Eickelberg $\mathrm{O}$. The impact of TGF- $\beta$ on lung fibrosis from targeting to biomarkers. Proc Am Thorac Soc 2012; 9: 111-116.

55 Gangwar I, Sharma NK, Panzade G, et al. Detecting the molecular system signatures of idiopathic pulmonary fibrosis through integrated genomic analysis. Sci Rep 2017; 7: 1554.

56 Knudsen L, Ruppert C, Ochs M. Tissue remodelling in pulmonary fibrosis. Cell Tissue Res 2017; 367: 607-626.

57 Hardie WD, Hagood JS, Dave V, et al. Signaling pathways in the epithelial origins of pulmonary fibrosis. Cell Cycle 2010; 9: 2769-2776.

58 O'Dwyer DN, Norman KC, Xia M, et al. The peripheral blood proteome signature of idiopathic pulmonary fibrosis is distinct from normal and is associated with novel immunological processes. Sci Rep 2017; 7: 46560.

59 Cox G, Kable E. Second-harmonic imaging of collagen. Methods Mol Biol 2006; 319: 15-35.

60 Liu W, Raben N, Ralston E. Quantitative evaluation of skeletal muscle defects in second harmonic generation images. J Biomed Opt 2013; 18: 026005.

$61 \mathrm{Yu} \mathrm{C-H,} \mathrm{Langowitz} \mathrm{N,} \mathrm{Wu} \mathrm{HY,} \mathrm{et} \mathrm{al.} \mathrm{Measuring} \mathrm{microtubule} \mathrm{polarity} \mathrm{in} \mathrm{spindles} \mathrm{with} \mathrm{second-harmonic}$ generation. Biophys J 2014; 106: 1578-1587.

62 Kuhn C, Boldt J, King TE, et al. An immunohistochemical study of architectural remodeling and connective tissue synthesis in pulmonary fibrosis. Am Rev Respir Dis 1989; 140: 1693-1703.

63 Hansen NUB, Karsdal MA, Brockbank S, et al. Tissue turnover of collagen type I, III and elastin is elevated in the PCLS model of IPF and can be restored back to vehicle levels using a phosphodiesterase inhibitor. Respir Res 2016; 17: 76.

64 Han S, Li YY, Chan BP. Protease inhibitors enhance extracellular collagen fibril deposition in human mesenchymal stem cells. Stem Cell Res Ther 2015; 6: 197.

65 Dancer RCA, Wood AM, Thickett DR. Metalloproteinases in idiopathic pulmonary fibrosis. Eur Respir J 2011; 38: 1461-1467.

66 Adams TS, Schupp JC, Poli S, et al. Single-cell RNA-seq reveals ectopic and aberrant lung-resident cell populations in idiopathic pulmonary fibrosis. Sci Adv 2020; 6: eaba1983.

67 Habermann AC, Gutierrez AJ, Bui LT, et al. Single-cell RNA sequencing reveals profibrotic roles of distinct epithelial and mesenchymal lineages in pulmonary fibrosis. Sci Adv 2020; 6: eaba1972.

68 Travaglini KJ, Nabhan AN, Penland L, et al. A molecular cell atlas of the human lung from single-cell RNA sequencing. Nature 2020; 587: 619-625.

69 Zhang Q, He Y, Luo N, et al. Landscape and dynamics of single immune cells in hepatocellular carcinoma. Cell 2019; 179: 829-845.e20.

70 Stegmayr J, Alsafadi HN, Langwinski W, et al. Isolation of high yield and quality RNA from human precision-cut lung slices for RNA-sequencing and computational integration with larger patient cohorts. Am J Physiol Lung Cell Mol Physiol 2021; 320: L232-L240.

71 Gabr SA, Alghadir AH, Sherif YE, et al. Hydroxyproline as a biomarker in liver disease. In: Patel V, Preddy V, eds. Biomarkers in Liver Disease. Biomarkers in Disease Methods: Methods, Discoveries and Applications. Dordecht, Springer, 2017.

72 Hewitson TD, Smith ER, Samuel CS, et al. Qualitative and quantitative analysis of fibrosis in the kidney. Nephrology 2014; 19: 721-726. 
73 Puthawala K, Hadjiangelis N, Jacoby SC, et al. Inhibition of integrin $\alpha v \beta 6$, an activator of latent transforming growth factor- $\beta$, prevents radiation-induced lung fibrosis. Am J Respir Crit Care Med 2008; 177: 82-90.

74 Page-McCaw A, Ewald AJ, Werb Z. Matrix metalloproteinases and the regulation of tissue remodelling. Nat Rev Mol Cell Biol 2009; 8: 221-233.

75 Zank DC, Bueno M, Mora AL, et al. Idiopathic pulmonary fibrosis: aging, mitochondrial dysfunction, and cellular bioenergetics. Front Med 2018; 5: 10.

76 Jones MG, Fabre A, Schneider P, et al. Three-dimensional characterization of fibroblast foci in idiopathic pulmonary fibrosis. JCl Insight 2016; 1: e86375.

77 Arora PD, Narani N, McCulloch CAG. The compliance of collagen gels regulates transforming growth factor- $\beta$ induction of $\alpha$-smooth muscle actin in fibroblasts. Am J Pathol 1999; 154: 871-882.

78 Wipff P-J, Rifkin DB, Meister J-J, et al. Myofibroblast contraction activates latent TGF- $\beta 1$ from the extracellular matrix. J Cell Biol 2007; 179: 1311-1323.

79 Zscheppang K, Berg J, Hedtrich S, et al. Human pulmonary 3D models for translational research. Biotechnol J 2018; 13: 1700341.

80 Desai O, Winkler J, Minasyan M, et al. The role of immune and inflammatory cells in idiopathic pulmonary fibrosis. Front Med 2018; 5: 43. 